\title{
Impact of Inter- and Intra-Regional Coordination in Markets With a Large Renewable Component
}

Delikaraoglou, Stefanos; Morales González, Juan Miguel; Pinson, Pierre

Published in:

IEEE Transactions on Power Systems

Link to article, DOI:

10.1109/TPWRS.2016.2530981

Publication date:

2016

Document Version

Peer reviewed version

Link back to DTU Orbit

Citation (APA):

Delikaraoglou, S., Morales González, J. M., \& Pinson, P. (2016). Impact of Inter- and Intra-Regional

Coordination in Markets With a Large Renewable Component. IEEE Transactions on Power Systems, 31(6), 5061-5070. https://doi.org/10.1109/TPWRS.2016.2530981

\section{General rights}

Copyright and moral rights for the publications made accessible in the public portal are retained by the authors and/or other copyright owners and it is a condition of accessing publications that users recognise and abide by the legal requirements associated with these rights.

- Users may download and print one copy of any publication from the public portal for the purpose of private study or research.

- You may not further distribute the material or use it for any profit-making activity or commercial gain

- You may freely distribute the URL identifying the publication in the public portal 


\title{
Impact of Inter- and Intra-Regional Coordination in Markets With a Large Renewable Component
}

\author{
Stefanos Delikaraoglou, Student Member, IEEE, Juan M. Morales, Member, IEEE, and \\ Pierre Pinson, Senior Member, IEEE
}

\begin{abstract}
The establishment of the single European day-ahead market has accomplished a crucial step towards the spatial integration of the European power system. However, this new arrangement does not consider any intra-regional coordination of dayahead and balancing markets and thus may become counterproductive or inefficient under uncertain supply, e.g., from weatherdriven renewable power generation. In the absence of a specific target model for the common balancing market in Europe, we introduce a framework to compare different coordination schemes and market organizations. The proposed models are formulated as stochastic equilibrium problems and compared against an optimal market setup. The simulation results reveal significant efficiency loss in case of partial coordination and diversity of market structure among regional power systems.
\end{abstract}

Index Terms-Market coupling, TSO coordination, stochastic complementarity models, generalized Nash equilibrium (GNE), electricity markets.

\section{NOTATION}

\section{A. Indices and sets:}

$A \quad$ Set of regions

$N \quad$ Set of system buses

I Set of dispatchable units

$J \quad$ Set of wind power units

$L \quad$ Set of transmission lines

$\Omega \quad$ Set of wind power scenarios

$\mathcal{M}_{n}^{I} \quad$ Set of dispatchable units located at bus $n$

$\mathcal{M}_{n}^{J} \quad$ Set of wind power units located at bus $n$

$\mathcal{M}_{a}^{N} \quad$ Set of buses located in region $a$

$\mathcal{M}_{a}^{I} \quad$ Set of dispatchable units located in region $a$

\section{B. Parameters:}

$D_{n} \quad$ Demand at node $n$ [MW]

$C_{i} \quad$ Day-ahead offer price of unit $i$ [\$/MWh]

Manuscript received September 14, 2015; revised January 02, 2016; accepted February 03, 2016. The work of S. Delikaraoglou was supported by Energinet.dk in support of ERA-Net SmartGrids through project BPES-Balancing Power in the European System (No. 2010-1-10816). The work of J. M. Morales and P. Pinson was supported in part by the Danish Strategic Council for Strategic Research through the project $5 \mathrm{~s}$ - Future Electricity Markets (no. 12-132636/DSF). Paper no. TPWRS-01302-2015.

S. Delikaraoglou and P. Pinson are with the Department of Electrical Engineering, Technical University of Denmark, Lyngby 2800, Denmark (e-mail: stde@dtu.dk; ppin@dtu.dk).

J. M. Morales is with the Department of Applied Mathematics and Computer Science, Technical University, Lyngby 2800, Denmark (e-mail: jmmgo@dtu. $\mathrm{dk})$.

$\begin{array}{ll}C_{i}^{+} & \text {Up regulation offer price of unit } i[\$ / \mathrm{MWh}] \\ C_{i}^{-} & \text {Down regulation offer price of unit } i[\$ / \mathrm{MWh}] \\ C^{\mathrm{sh}} & \text { Value of involuntarily shed load [\$/MWh] } \\ P_{i}^{\max } & \text { Capacity of dispatchable unit } i[\mathrm{MW}] \\ W_{j}^{\max } & \text { Capacity of wind power unit } j[\mathrm{MW}] \\ \bar{W}_{j} & \text { Forecast mean of wind power production [MW] } \\ W_{j \omega} & \text { Wind power realization in scenario } \omega[\mathrm{MW}] \\ \pi_{\omega} & \text { Probability of occurrence of scenario } \omega \\ R_{i}^{+} & \text {Maximum up regulation provided by unit } i \text { [MW] } \\ R_{i}^{-} & \text {Maximum down regulation provided by unit } i \\ f_{n r}^{\max } & \text { [MW] } \\ B_{n r} & \text { Maximum capacity of line }(n, r)[\mathrm{MW}] \\ \Phi_{n r}^{a} & \text { Absolute value of the susceptance of line }(n, r)\end{array}$

C. Variables:

$\hat{\delta}_{n} \quad$ Voltage angle at node $n$ at the day-ahead stage [rad]

$\tilde{\delta}_{n \omega} \quad$ Voltage angle at node $n$ in scenario $\omega$ [rad]

$p_{i} \quad$ Day-ahead schedule of dispatchable unit $i$ [MW]

$w_{j} \quad$ Day-ahead schedule of wind power unit $j$ [MW]

$\bar{r}_{i}^{+} \quad$ Up reserve capacity scheduled for unit $i$ [MW]

$\bar{r}_{i}^{-} \quad$ Down reserve capacity scheduled for unit $i$ [MW]

$r_{i \omega}^{+} \quad$ Up regulation provided by unit $i$ in scenario $\omega$ [MW]

$r_{i \omega}^{-} \quad$ Down regulation provided by unit $i$ in scenario $\omega$ [MW]

$w_{j \omega}^{\text {spill }} \quad$ Wind power spilled by unit $j$ in scenario $\omega[\mathrm{MW}]$

$l_{n \omega}^{\mathrm{sh}} \quad$ Load shedding at node $n$ in scenario $\omega$ [MW]

Variables augmented with the superscript $a$ represent the decisions of the operator in charge of the corresponding region. The symbols $p r_{A}^{\circledast}, p r_{B}^{\circledast}$ denote the valuation of the arbitrary resource $\circledast$ by operators A and B, respectively. Similarly, $q_{A}, q_{B}$ denote the subset of system resources located in the corresponding regions $\mathrm{A}$ and $\mathrm{B}$.

\section{INTRODUCTION}

$\mathbf{T}$ HE last years have seen a substantial restructuring of the European power system regarding both the generation portfolio and the market architecture. Renewable energy sources (RES) have gained a leading role on the supply-side, Digital Object Identifier 10.1109/TPWRS.2016.2530981 
while the establishment of the Internal Electricity Market (IEM) pursued the spatial integration of the regional day-ahead markets. This inter-regional integration of day-ahead markets has enabled access to a larger pool of generating resources and exploits the arbitrage opportunities over the European network to optimize social welfare through enhanced cross-border competition and improved market liquidity. According to the 'Target Model' for the European market integration, day-ahead clearing follows a zonal pricing scheme, where transmission capacity among zones is calculated according to either flow-based methods in case of highly meshed grids and strong interdependencies between the interconnections or the Available Transfer Capacity (ATC) approach for less meshed networks [1]. This reduced grid representation, where internal network is treated as a copper plate, may result into infeasible real-time power flows due to transmission congestion. Nevertheless, recent literature [2], [3] proposes alternative approaches to account also for the intra-zonal network feasibility, based on a centralized market-splitting algorithm that integrates diverse market designs, i.e., power pools or power exchanges.

Despite the spatial coupling of day-ahead markets operated by power exchanges, balancing operation remains still a responsibility of regional Transmission System Operators (TSOs). During real-time operation, TSOs must take corrective actions to guarantee network feasibility and cover any imbalances from the initial schedule. This geographically segmented design hinders access to cheaper cross-border balancing resources and thus it aggravates the scope for exercising market power in cases of low market liquidity. Moreover, internal balancing resources might be over-utilized since some potential gains from reduced prediction errors due to spatial smoothing effects of stochastic in-feed are not leveraged. In this respect, [4] shows that the integration of the Nordic and German balancing markets can reduce annual costs up to $€ 400$ million, while [5] estimates a reduction of balancing costs up to $50 \%$ from the establishment of a common merit-order list between the Dutch, German and Nordic markets. In addition, [6] reports 40\% less re-dispatch in the integrated Northern European power market in case of system-wide regulating reserve procurement as well as $25 \%$ reduction in reserves activation due to system-wide netting of imbalances. A similar analysis is performed in [7], showing a potential cost reduction of $40 \%$ by the implementation of a cross-border balancing mechanism between Belgium and the Netherlands. On the European level, [8] estimates the benefits from balancing market integration to $€ 1.3$ billion per year due to potential imbalance netting and exchange of lower cost balancing power.

Apart from the limited coordination in space, the sequential operation of the current market architecture does not allow for any inter-temporal hedging to deal with the increasing uncertainty of variable and partially predictable RES, e.g., wind power, resulting in diminished overall market efficiency. In order to enable the coupling of day-ahead and balancing market stages, recent research [9]-[12] focuses on market-clearing models that jointly optimize day-ahead dispatch and real-time reserve deployment within a stochastic programming framework. This integrated approach is able to capture the dependency of balancing actions on day-ahead decisions and thus reduce total operating costs.

Nonetheless, the implementation of an ideal market design, which integrates energy and transmission both in time and space, requires a pan-European market operator/TSO that has full information on the spatio-temporal structure of forecast errors and a complete overview of the system. However, regional TSOs are hesitant to forfeit some of their autonomy to a central authority given the significant differences that exist in balancing operations, control concepts and local resources. In the absence of a specific target model for the integration of regional balancing markets, different market arrangements with varying levels of harmonization and coordination between TSOs may arise before the establishment of a pan-European balancing market [13]. A comprehensive study on the different design elements in six short-term, i.e., intraday and real-time, European power markets is presented in [14]. In this regard, generalized Nash equilibrium (GNE) problems provide a natural framework to model the interaction of players that share a common strategy set. For instance, [15] formulates a GNE model to study different degrees of coordination in congestion management among neighboring TSOs. This model is applied on an illustrative power system in [16], [17] showing the detrimental effects of partial coordination and market incompleteness on re-dispatching costs, while [18] follows a similar approach to study the effect of imperfect coordination among the four German TSOs which results in expensive remedial actions.

This paper aims at appraising the benefits from the spatio-temporal coupling of the European electricity markets compared to plausible decentralized organizations with imperfect coordination among neighboring regions. In this regard, we provide a methodological framework to model varying levels of coordination both in space and in time based on a stochastic mixed complementarity problem (MCP) formulation. The proposed framework can accommodate several features of possible market arrangements such as limited access to cross-border balancing resources and different valuation of the shared transmission capacity. In light of an inefficient energy-only market in case of partial coordination, we propose a remedial reserve capacity mechanism that brings the market outcome close to the ideal setup of full coordination.

The rest of the paper is organized as follows. Section II describes the different degrees of intra- and inter-regional coordination and their modeling approach from an economic and mathematical perspective, while the detailed model formulation is provided in Section III. Section IV presents and discusses results of an illustrative case study performed on a stylized six-bus electricity system. Finally, Section V concludes the paper.

\section{BASIC COORDINATION CONCEPTS}

In this work, we study the impact of different degrees of coordination both in time (intra-regional) and in space (inter-regional) domains, as shown in Fig. 1.

On the temporal dimension of market operation, we model the extreme cases of imperfect and full intra-regional coordination by employing a sequential and a stochastic integrated market- 


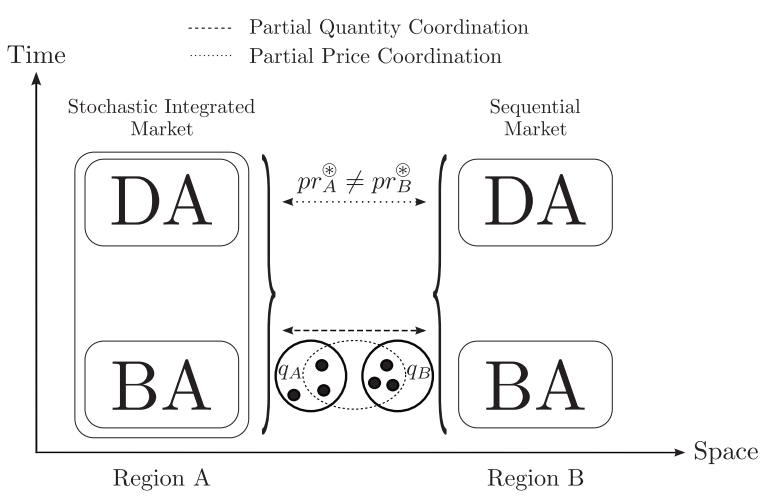

Fig. 1. Intra- and inter-regional coordination arrangements. DA: Day-ahead market. BA: Balancing market.

clearing approach, respectively. The sequential setup resembles the existing European market architecture, where a day-ahead auction takes place 12-36 hours prior to actual energy delivery to produce an initial schedule for generation and consumption levels. To cope with any real-time energy imbalances, an independent balancing market is organized for the activation of regulating power. This mechanism perceives a completely deterministic view of the uncertainties involved in power system operation, e.g., stochastic wind power generation, and thus it prevents any intra-regional coordination between the day-ahead and balancing trading floors. On the contrary, a market-clearing model which co-optimizes the energy dispatch and the anticipated reserve deployment with a fully probabilistic view of uncertainty, attains perfect intra-regional coordination. Nonetheless, this market setup suffers from a fundamental design flaw: marginal prices do not always guarantee cost recovery for flexible producers [10], [19]. Hence, the application of this market architecture here is purely instrumental and used only for the definition of an optimal benchmark for intra-regional coordination.

In the inter-regional domain, full spatial coordination is achieved when regional operators ${ }^{1}$ cooperate to jointly optimize their systems, offering all their resources in a common pool and exchanging complete information about their domestic network. On the other hand, imperfect coordination between multiple operators may arise in form of partial quantity or price coordination. Partial quantity coordination emerges in case of a segmented market of cross-border resources, i.e., when some internal resources are excluded from the common pool, as shown in Fig. 1, where only a subset of resources $q_{A}$ is offered in the common market, e.g., due to different reliability criteria. Throughout this work, we consider that all system resources are accessible from all regional operators during the day-ahead stage, following the paradigm of the European Internal Electricity Market. On the contrary, we apply the concept of partial quantity coordination on the balancing stage, where each operator can access only the subset of system resources that are physically located in its region.

\footnotetext{
${ }^{1}$ We couple the entities of market operator and TSO under the term 'operator', despite their distinguished scope pertaining to the day-ahead and balancing markets, respectively.
}

Another source of imperfect coordination is market incompleteness [20]. A complete market suggests that all constraints are traded at a single price which reflects the common valuation of each product from all agents. However, in case of partial price coordination (or a completely missing market for some services), agents with different willingness to pay for a certain resource face a price gap due to lack of arbitrage opportunities that prevent price convergence [21]. For example, in Fig. 1 the arbitrary resource $\circledast$ (e.g., a transmission line or generation capacity) is valued at different prices $p r_{A}^{\circledast} \neq p r_{B}^{\circledast}$ from each operator. This leads to a Pareto-inefficient resource allocation since all agents could improve their payoff by jointly optimizing their decisions [22].

From an economic perspective, imperfect coordination among various players relates to the notion of GNE. This is the equilibrium of a non-cooperative game where both the payoff and the set of each player's constraints depends on rival players' actions. In the context of power systems, operators in adjacent regions interact on their strategy sets through the shared physical constraints imposed by the underlying network and the limits of the available resources.

From a mathematical viewpoint, a GNE problem leads to a Quasi-Variational Inequality (QVI) model which, in the general case, admits multiple solutions that arise from different valuation of the shared constraints, i.e., each agent may have different dual variables for each shared constraint. In order to obtain a numerically tractable solution, the original QVI problem is reduced to a Variational Inequality (VI) problem, using the solution concept of normalized equilibrium [23]. Finally, this VI problem can be recast as an equivalent MCP as described in Section III.

Similar methods to explore the multiple solutions to QVI problems are proposed in [24], whereas [25] formulates an iterative algorithm based on disjunctive constraints to identify different equilibria, at the expense, though, of higher computational cost. The normalized equilibrium technique is also used in [26] to analyze cooperative game theory in the context of electricity markets as well as in [27] for the analysis of renewable portfolio standards in the presence of coupling regulatory constraints among different electricity producers.

\section{Modeling InTRA- AND INTER-REgIONAL COORDINATION}

\section{A. Assumptions}

The mathematical formulation of market-clearing models (1)-(2) and (3) build on the following set of assumptions.

1) The only source of uncertainty considered is stochastic wind power generation while system load and 'firm' resource availability can be perfectly forecast. However, additional uncertainties such as demand variations or equipment failures can be integrated in the market-clearing in a similar way.

2) Stochastic wind power production is modeled by a finite set of scenarios. This probabilistic information is centrally obtained by the operator to capture the spatial dependence structure of the forecast errors. In addition, we assume access to the correct description of the predictive densities of stochastic power production, i.e., there is no mismatch 
between the 'estimated' and the 'realized' distributions of wind energy generation. We leave the topic of forecast imperfection for future research.

3) Demand-side is assumed to be completely inelastic, with a sufficiently large value of lost load. Hence, the social welfare maximization is equivalent to minimization of the power system operational cost.

4) The day-ahead market is settled on independent hourly single-period auctions and thus no inter-temporal constraints, e.g., ramping rates, are taken into account. Nonetheless, ramping constraints can be incorporated in the proposed framework, extending the formulation to a multi-period setup. In addition, no intra-day energy trading is considered, justified by the low liquidity of these markets [28].

5) The transmission network is modeled with a lossless DC approximation.

6) Assuming null power production costs, the price offer and the spillage cost of wind power is set equal to zero.

7) In accordance with the European market paradigm, conventional generators internalize any discrete on-off commitment decisions and we further assume that they offer their full capacity into the market with linear cost functions. This preserves the linear and convex formulation of the market-clearing models and permits to integrate the market-clearing models in a complementarity problem through their Karush-Kuhn-Tucker (KKT) conditions.

In the subsequent formulations, the vector of dual variables associated with each set of constraints is indicated after a colon. For the sake of clarity, we use the symbols $\mu$ and $\lambda$, with appropriate indices, to denote the duals of inequality and equality constraints, respectively.

\section{B. Intra-Regional Coordination}

1) Sequential Market (SeqM): The sequential market setup comprises the day-ahead and balancing trading floors which are cleared independently by solving models (1) and (2), respectively. The operator determines the optimal day-ahead dispatch $\left(p_{i}^{*}, \forall i ; w_{j}^{*}, \forall j\right)$ through the following optimization problem.

$$
\underset{\Xi^{\mathrm{DA}}}{\operatorname{Min}} \sum_{i \in I} C_{i} p_{i}
$$

subject to

$$
\begin{aligned}
& \sum_{i \in \mathcal{M}_{n}^{I}} p_{i}+\sum_{j \in \mathcal{M}_{n}^{J}} w_{j}-D_{n} \\
& -\sum_{r:(n, r) \in L} B_{n r}\left(\hat{\delta}_{n}-\hat{\delta}_{r}\right)=0: \hat{\lambda}_{n}, \forall n \\
& p_{i} \leq P_{i}^{\max }: \mu_{i}^{P^{\max }}, \forall i \\
& w_{j} \leq \bar{W}_{j}: \mu_{j}^{W^{\max }}, \forall j \\
& B_{n r}\left(\hat{\delta}_{n}-\hat{\delta}_{r}\right) \leq f_{n r}^{\max }: \hat{\mu}_{n r}^{f^{\max }}, \forall(n, r) \in L \\
& \hat{\delta}_{n}=0: \hat{\lambda}^{\mathrm{ref}}, n: \operatorname{ref} \\
& p_{i} \geq 0, \forall i ; w_{j} \geq 0, \forall j ; \hat{\delta}_{n} \text { free, } \forall n \backslash n: \text { ref }
\end{aligned}
$$

where $\Xi^{\mathrm{DA}}=\left\{p_{i}, \forall i ; w_{j}, \forall j ; \hat{\delta}_{n}, \forall n\right\}$ is the set of primal optimization variables of the day-ahead market-clearing problem. The objective function (1a) represents the day-ahead cost of power system operation to be minimized. The equality constraint (1b) enforces the power balance at every node of the system. Constraints (1c) represent the capacity limits of conventional generators, while constraints (1d) set the upper bound to wind power dispatch equal to the conditional expectation of the wind power stochastic process. The power flows over the network are restricted by the transmission capacity limits in (1e) and equation (1f) fixes the voltage angle at the reference node equal to zero. Finally, constraints $(1 \mathrm{~g})$ are variable declarations.

In real-time operation, when the uncertainty realization $W_{j \omega^{\prime}}$ is known, any deviations from the day-ahead schedule have to be balanced by appropriate re-dispatch actions given as the solution of the following optimization problem.

$$
\operatorname{Min}_{\Xi^{\mathrm{RT}}} \sum_{i \in I}\left(C_{i}^{+} r_{i \omega^{\prime}}^{+}-C_{i}^{-} r_{i \omega^{\prime}}^{-}\right)+\sum_{n \in N} C^{\mathrm{sh}} l_{n \omega^{\prime}}^{\mathrm{sh}}
$$

subject to

$$
\begin{aligned}
& \sum_{i \in \mathcal{M}_{n}^{I}}\left(r_{i \omega^{\prime}}^{+}-r_{i \omega^{\prime}}^{-}\right)+\sum_{j \in \mathcal{M}_{n}^{J}}\left(W_{j \omega^{\prime}}-w_{j}^{*}-w_{j \omega^{\prime}}^{\mathrm{spill}}\right)+l_{n \omega^{\prime}}^{\mathrm{sh}} \\
& +\sum_{r:(n, r) \in L} B_{n r}\left(\hat{\delta}_{n}^{*}-\tilde{\delta}_{n \omega^{\prime}}-\hat{\delta}_{r}^{*}+\tilde{\delta}_{r \omega^{\prime}}\right)=0: \tilde{\lambda}_{n \omega^{\prime}}, \forall n(2 \mathrm{~b}) \\
& r_{i \omega^{\prime}}^{+} \leq P_{i}^{\mathrm{max}}-p_{i}^{*}: \mu_{i \omega^{\prime}}^{\mathrm{PR}^{+}}, \forall i \\
& r_{i \omega^{\prime}}^{-} \leq p_{i}^{*}: \mu_{i \omega^{\prime}}^{\mathrm{PR}}, \forall i \\
& r_{i \omega^{\prime}}^{+} \leq R_{i}^{+}: \mu_{i \omega^{\prime}}^{\mathrm{R}^{+}}, \forall i \\
& r_{i \omega^{\prime}}^{-} \leq R_{i}^{-}: \mu_{i \omega^{\prime}}^{\mathrm{R}}, \forall i \\
& w_{j \omega^{-}}^{\mathrm{spill}} \leq W_{j \omega^{\prime}}: \mu_{j \omega^{\prime}}^{w^{\mathrm{spill}}}, \forall j \\
& l_{n \omega^{\prime}}^{\mathrm{sh}} \leq D_{n}: \mu_{n \omega^{\prime}}^{l^{\mathrm{sh}}}, \forall n \\
& B_{n r}\left(\tilde{\delta}_{n \omega^{\prime}}-\tilde{\delta}_{r \omega^{\prime}}\right) \leq f_{n r}^{\max }: \tilde{\mu}_{n r \omega^{\prime}}^{f^{\max }, \forall(n, r) \in}(2 \mathrm{c}) \\
& \tilde{\delta}_{n \omega^{\prime}}=0: \tilde{\lambda}_{\omega^{\prime}}^{\mathrm{ref}}, n: \text { ref } \\
& r_{i \omega^{\prime}}^{+}, r_{i \omega^{\prime}}^{-} \geq 0, \forall i ; w_{j \omega^{\prime}}^{\mathrm{spill}} \geq 0, \forall j ; \\
& l_{n \omega^{\prime}}^{\mathrm{sh}} \geq 0, \forall n ; \tilde{\delta}_{n \omega^{\prime}} \text { free, } \forall n \backslash n: \text { ref }
\end{aligned}
$$

where $\Xi^{\mathrm{RT}}=\left\{r_{i \omega^{\prime}}^{+}, r_{i \omega^{\prime}}^{-}, \forall i ; w_{j \omega^{\prime}}^{\text {spill }}, \forall j ; l_{n \omega^{\prime}}^{\text {sh }}, \forall n ; \tilde{\delta}_{n \omega^{\prime}}, \forall n\right\}$ is the set of primal optimization variables of the balancing marketclearing problem. Notice that all the day-ahead variables enter the problem as parameters. The re-dispatch actions, i.e., up- and down-regulation, wind spillage and load shedding constitute the set of available regulation services. The objective function (2a) to be minimized is the cost of re-dispatch actions in order to re-establish the nodal power balance enforced by constraints (2b). Constraints (2c) and (2d) bind the up and down reserve activation from every conventional unit according to its capacity and the day-ahead dispatch, while constraints (2e) and (2f) enforce the corresponding offer limits specified by each generator for up- and down-regulation, respectively. Similarly, the re-dispatch actions of wind spillage and load shedding are bounded by the actual wind power production and the nodal demand through $(2 \mathrm{~g})$ and $(2 \mathrm{~h})$, respectively. Constraints (2i) guarantee the network feasibility after the deployment of reserves and equation (2j) fixes the voltage angle of the reference node to zero. The variable declarations of model (2) are included in the set of constraints (2k). 
2) Stochastic Integrated Market (StochM): The stochastic integrated market is formulated as a two-stage stochastic programming problem (3) in which day-ahead dispatch decisions anticipate the balancing recourse actions over a plausible range of uncertainty. For the sake of brevity, we present a compact formulation of model (3) emphasizing its main differences compared to the sequential approach.

$$
\underset{\Xi^{\mathrm{ST}}}{\operatorname{Min}} \sum_{i \in I} C_{i} p_{i}+\sum_{\omega \in \Omega} \pi_{\omega}\left[\sum_{i \in I}\left(C_{i}^{+} r_{i \omega}^{+}-C_{i}^{-} r_{i \omega}^{-}\right)+\sum_{n \in N} C^{\mathrm{sh}} l_{n \omega}^{\mathrm{sh}}\right]
$$

subject to

$$
\begin{aligned}
& \text { constraints }(1 \mathrm{~b})-(1 \mathrm{c}),(1 \mathrm{e})-(1 \mathrm{~g}) \\
& w_{j} \leq W_{j}^{\max }: \mu_{j}^{W^{\max }}, \forall j \\
& \text { constraints }(2 \mathrm{~b})-(2 \mathrm{k}), \forall \omega
\end{aligned}
$$

where $\Xi^{\mathrm{ST}}=\left\{p_{i}, r_{i \omega}^{+}, r_{i \omega}^{-}, \forall i, \forall \omega ; w_{j}, w_{j \omega}^{\mathrm{spill}}, \forall j, \forall \omega ; l_{n \omega}^{\mathrm{sh}}, \forall n\right.$, $\left.\forall \omega ; \hat{\delta}_{n}, \tilde{\delta}_{n \omega}, \forall n, \forall \omega\right\}$ is the set of primal optimization variables of the stochastic integrated market model. Unlike the conventional dispatch model (1)-(2), which separates completely the day-ahead and balancing operations, the stochastic integrated market accounts for the interaction of these stages through the expectation of the balancing costs in the objective function (3a) and the real-time constraints (3d). Notice that real-time constraints are formulated for all $\omega \in \Omega$, whereas the day-ahead dispatch decisions enter the set of (3d) as variables instead of fixed parameters. This implicit link between the two stages permits to pre-position, even out of merit-order, the flexible resources in a way that improves the overall response to variations and uncertainty of stochastic generation. Moreover, observe that, the day-ahead dispatch of stochastic generation may take values within the whole interval of the installed capacity (constraint (3c)) depending on the structure of forecast errors and the asymmetry of regulating costs.

\section{Inter-Regional Coordination}

To study different degrees of inter-regional coordination, we consider a multi-regional power system where each region $a \in$ $A$ is managed by an independent operator. In this setup, full coordination is achieved when all operators cooperate in order to minimize the total system cost by fully sharing the resources and technical information of their jurisdiction both in the dayahead and real-time stages. In practice, this setup is equivalent to a fully centralized organization where market-clearing is performed by a single entity. However, a decentralized organizational structure, where each regional operator seeks to minimize its own internal system cost, may not fulfill the prerequisites of the above ideal paradigm. Following the discussion of Section II, this operational scheme is modeled as a stochastic $\mathrm{MCP}$, where the KKT conditions for each operator's optimization problem are simultaneously solved. This modeling framework allows to consider regional operators that follow either similar or different market designs, in particular the 'naive' sequential approach or the 'smart' stochastic integrated marketclearing. The complete formulation of KKT optimality conditions is provided in the Appendix.
We first focus on imperfect quantity coordination only in the balancing stage, assuming that operators fully cooperate in the day-ahead stage, similarly to the existing IEM organization where all regions have access to a common system-wide pool of generation resources. Mathematically, this is implemented by replacing constraints (1c)-(1d) by (4a)-(4b), where the day-ahead dispatch variables for conventional and wind power units are now augmented with the superscript ' $a$ ' to denote the decisions of the corresponding operator. Note that in principle these are shared constraints between all operators. However, the establishment of a common day-ahead market implies that there exists only a single price for each resource, i.e., the corresponding dual variables are equal for every operator.

$$
\begin{aligned}
& \sum_{a \in A} p_{i}^{a} \leq P_{i}^{\max }: \mu_{i}^{P^{\max }}, \forall i \\
& \sum_{a \in A} w_{j}^{a} \leq \bar{W}_{j}: \mu_{j}^{W^{\max }}, \forall j
\end{aligned}
$$

Similarly, if the stochastic integrated market is employed, constraint (4b) replaces (3c) and thus its right-hand side is set equal to $W_{j}^{\max }$.

On the other hand, we assume a completely segmented balancing market where each operator has no access to cross-border resources during real-time operation. From a modeling perspective, this setup requires the replacement of reserve deployment constraints (2c)-(2d) by (5a)-(5b), which are however included only in the optimization problem of the operator who controls the region where the corresponding resource is located. Hence, these are not shared constraints, even though they include day-ahead dispatch decisions from other regional operators, and thus there exists per se a single vector of dual variables. A similar logic applies for the rest of re-dispatch actions which are solely performed by each regional operator, i.e., constraints $(2 \mathrm{e})-(2 \mathrm{~h})$. Observe that this set of constraints' substitution applies both to the sequential and the stochastic integrated market-clearing models.

$$
\begin{aligned}
& \sum_{a \in A} p_{i}^{a}+r_{i \omega}^{+} \leq P_{i}^{\mathrm{max}}: \mu_{i \omega}^{\mathrm{PR}^{+}}, \forall i \in \mathcal{M}_{a}^{I}, \forall \omega \\
& r_{i \omega}^{-}-\sum_{a \in A} p_{i}^{a} \leq 0: \mu_{i \omega}^{\mathrm{PR}^{-}} \forall i \in \mathcal{M}_{a}^{I}, \forall \omega
\end{aligned}
$$

Extending our framework into the concept of partial price coordination, we consider an incomplete market of transmission resources during balancing operation. This organizational arrangement implies that each operator is responsible to maintain the real-time power balance only in its internal network, while it has no information on the domestic grid of the neighboring regions, i.e., there is no market of internal transmission resources. Nonetheless, adjacent power systems still interact via the shared power flow constraints of the tie-lines which are influenced by the re-dispatch actions in both regions due to the underlying network topology. Hence, the transmission capacity constraints of an interconnection $(n, r)$, similar to (2i), are included in the optimization problems of both operators $a$ and $a^{\prime}$ who control the respective sending and receiving ends. As a result, there exist two distinct dual variables $\tilde{\mu}_{n r \omega}^{f^{\max } \mid a}$ and $\tilde{\mu}_{n r \omega}^{f^{\max } \mid a^{\prime}}$, reflecting the marginal valuation of each operator, which in the 
absence of a trading mechanism may not converge to a single price. In fact, this absence of a unique price for the linking constraints indicates an incomplete market of common resources and yields an under-determined system. The reason is that each player's strategic decisions influence the optimal choices of the rivals and thus the right-hand side of the shared constraints; consequently, the initial strategy has to be revised. This mathematically translates into a non-square system with more variables than equations, given that each shared constraint is complementary to two dual variables.

In order to obtain a well-defined model, [29] suggests to exogenously impose identical dual variables of the linking constraints for all players. In turn, this reduces the original GNE to a Nash equilibrium (NE) which can be modeled as a VI problem and reformulated as an equivalent $\mathrm{MCP}$, which can be readily solved. However, this numerical approach introduces an implicit coordination structure akin to a marketplace where all agents can trade their shared constraints at a common price. Considering though that we are mainly interested to model the lack of arbitrage opportunities in an incomplete market, we employ an alternative technique referred as normalized equilibrium [23]; that is, the dual variables of the shared constraints for different agents follow a predetermined linear relationship that imposes their proportional variation. This exogenous relationship reduces the degrees of freedom of the system but it prevents them from converging on the same price. A normalized equilibrium point for the tie-line capacity constraints (2i) is defined as:

$$
\tilde{\mu}_{n r \omega}^{f^{\max } \mid a}=\Phi_{n r}^{a} \circ \tilde{\mu}_{n r \omega}^{f^{\max }}, \forall(n, r) \in L, \forall \omega, \forall a,
$$

where $\circ$ denotes the element-by-element multiplication. The entries of matrix $\Phi_{n r}^{a}$ reflect the relative valuation of each line $(n, r)$ by the regional operator $a$ with respect to a common dual variable $\tilde{\mu}_{n r \omega}^{f^{\max }}$. Hence, an instance of equal $\Phi_{n r}^{a}$ elements for every operator indicates a complete market for congestion on line $(n, r)$ or a case of partial price coordination otherwise.

This method allows us to investigate distinct preferences of individual players on common resources, e.g., tie-line capacity. These preferences are expressed through the corresponding value of the parameter $\Phi_{n r}^{a}$, which reflects the perception of operator $a$ for the value of tie-line $(n, r)$. For instance, the operator of a poorly interconnected power system may rely more on a specific tie-line to ensure adequate balancing resources than the neighboring operator who is well interconnected with several other regions. Here, it should be emphasized that this mathematical implementation allows us to obtain some of the possibly multiple equilibria and does not guarantee exhaustive enumeration of all equilibrium points that may exist. Nonetheless, the focus of this work is mainly on the practical implications of incomplete markets as a form of imperfect coordination.

\section{Operating Reserves (OR)}

The stochastic integrated market of model (3) is an energy-only auction, since operational cost is proportional only to the amount of energy (day-ahead schedule and re-dispatch actions) actually delivered to the network. However, this mechanism can incorporate an additional capacity component, similar to the operating reserve markets, in order to simulate a regional electricity market that optimally draws local capacity resources from the common day-ahead pool according to the potential balancing needs to hedge against optimization errors [30]. To improve clarity, we underline the difference between operating reserves and regulation services (see also [9] and [10]). The former term refers to the procurement of reserve $\mathrm{ca}$ pacity which, in turn, is excluded from energy provision in the day-ahead market. On the other hand, regulation services relate to the actual deployment of operating reserves during real-time operation. These re-dispatch actions modify the day-ahead production/consumption schedule of the generators/loads and thus, provide an energy service to the system.

With the introduction of the operating reserves mechanism, the amount of capacity reserved in each regional market becomes an additional strategic decision variable of the corresponding operator, denoted by $\bar{r}_{i}^{+}$and $\bar{r}_{i}^{-}$for up and down reserve capacity, respectively. For this purpose, we add the dayahead constraints $(7 a)-(7 b)$ that bound the upward and downward reserve provision to the respective capacity offer and constraints (7c)-(7d) that define the complementary relationship of energy and reserve services.

$$
\begin{aligned}
& \bar{r}_{i}^{+} \leq R_{i}^{+}: \mu_{i}^{\mathrm{R}^{+}}, \forall i \\
& \bar{r}_{i}^{-} \leq R_{i}^{-}: \mu_{i}^{\mathrm{R}^{-}}, \forall i \\
& \sum_{a \in A} p_{i}^{a}+\bar{r}_{i}^{+} \leq P_{i}^{\mathrm{max}}: \mu_{i}^{\mathrm{PR}^{+}}, \forall i \\
& \bar{r}_{i}^{-}-\sum_{a \in A} p_{i}^{a} \leq 0: \mu_{i}^{\mathrm{PR}^{-}}, \forall i
\end{aligned}
$$

In the balancing counterpart, constraints (2c)-(2d) are removed, since they now become redundant, and constraints (2e)-(2f) are replaced by

$$
\begin{aligned}
& r_{i \omega}^{+} \leq \bar{r}_{i}^{+}: \mu_{i \omega}^{\bar{r}^{+}}, \forall i, \forall \omega \\
& r_{i \omega}^{-} \leq \bar{r}_{i}^{-}: \mu_{i \omega}^{\bar{r}^{-}}, \forall i, \forall \omega .
\end{aligned}
$$

Following a similar process, operating reserves can be integrated also in the sequential market-clearing model (1)-(2). However, this would require the definition of explicit reserve requirements, which is beyond the scope of this paper. In addition, we would like to underline that operating reserves are not perceived here as a tradable product with explicit reserve capacity costs, but as a tool to indicate divergent policies for sharing internal balancing resources. Nonetheless, flexible generators are allowed to submit regulation offers with price premiums that compensate the opportunity costs of the power capacity withdrawn from the day-ahead dispatch, following the approach of [9].

\section{CASE Study}

The main purpose of this section is to provide some qualitative insights regarding the effect of imperfect coordination between neighboring power systems. To this aim, we consider the three distinct organizational setups presented in Fig. 2, with varying degrees of coordination and/or market architecture. The 


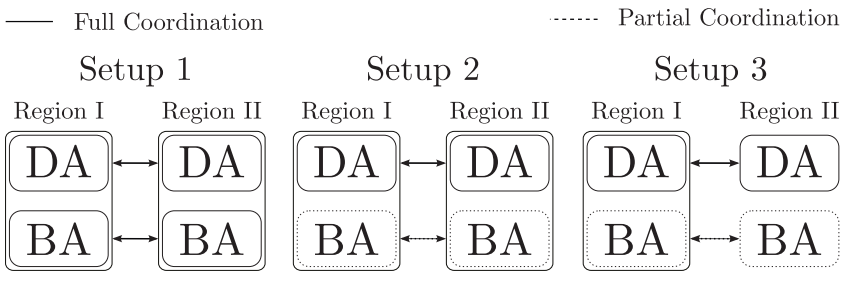

Fig. 2. Schematic representation of organizational setups with different market designs and degrees of coordination.

TABLE I

Model Definition for Organizational Setups

\begin{tabular}{llcc}
\hline Setup & \multicolumn{1}{c}{$\begin{array}{c}\text { Market } \\
\text { Organization }\end{array}$} & $\begin{array}{c}\text { Model } \\
\text { Definition }\end{array}$ & $\Phi_{n r}^{a}$ \\
\hline 1 & StochM & (3a)-(3d) & - \\
\hline 2A & StochM & $(9 \mathrm{a})-(9 \mathrm{~s})$ & $\Phi_{n r}^{a}=1$ \\
2B & StochM + OR & $(10 \mathrm{a})-(101)$ & $\Phi_{n r}^{a}=1$ \\
2C & StochM & $(9 \mathrm{a})-(9 \mathrm{~s})$ & $\Phi_{n r}^{a} \neq \Phi_{n r}^{a^{\prime}}$ \\
\hline 3 & Region I: StochM + OR & Stl: (10a)-(101) $\dagger$ & \multirow{2}{*}{$\Phi_{n r}^{a}=1$} \\
\hline
\end{tabular}

† Without variables pertaining to the balancing actions of region II.

${ }_{\ddagger}^{\ddagger}$ Solved for each scenario with the day-ahead variables fixed to $S t l$.

SeqM: Sequential Market. StochM: Stochastic Integrated Market. OR: Operating Reserves.

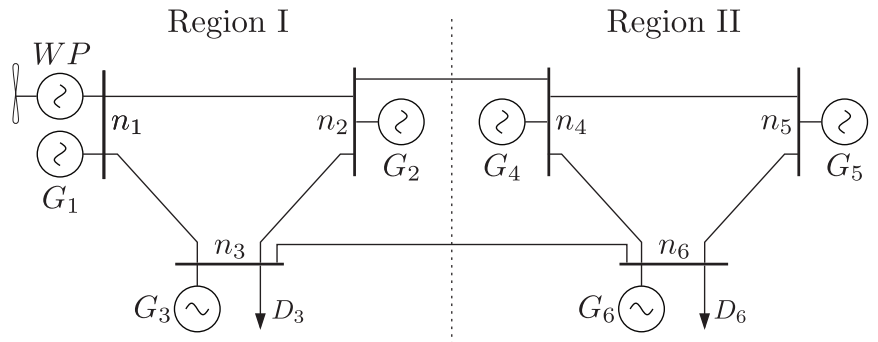

Fig. 3. Six-node power system.

model definition and the market architecture for each setup are summarized in Table I.

We consider the six-bus system depicted in Fig. 3 which comprises two regions. The transmission capacities and the reactances of all domestic lines are equal to 0.13 p.u. and $100 \mathrm{MW}$, respectively. The corresponding values for the tie-lines connecting region I to region II, are equal to 0.20 p.u. and $15 \mathrm{MW}$. Each region includes both flexible and inflexible conventional generators (G1 to G6) and one wind power plant (WP) is located at node 1 . The stochastic wind power generation is modeled using two scenarios, namely high $\left(\omega_{1}: 50 \mathrm{MW}\right)$ and low $\left(\omega_{2}\right.$ : $10 \mathrm{MW}$ ), with a probability of occurrence 0.6 and 0.4 , respectively. The quantity and the price offers of conventional units for the day-ahead and balancing markets are provided in Table II. In addition, two inelastic loads (D3 and D6) of $170 \mathrm{MW}$ and $190 \mathrm{MW}$, placed at nodes 3 and 6 , can be partially curtailed at a cost, $C^{\text {sh }}$, of $\$ 200 / \mathrm{MWh}$.

Setup 1: For our analysis, Setup 1 corresponds to perfect coordination both in the inter- and intra-regional domains. Here, both operators employ the stochastic integrated market design and fully cooperate in order to ensure system-wide network feasibility both in day-ahead and real-time operation with full ac-
TABLE II

Generator Data

\begin{tabular}{lcccccc}
\hline Unit $i$ & 1 & 2 & 3 & 4 & 5 & 6 \\
\hline$P_{i}^{\max }(\mathrm{MW})$ & 110 & 50 & 50 & 100 & 100 & 100 \\
$C_{i}(\$ / M W h)$ & 30 & 25 & 10 & 20 & 35 & 31 \\
$R_{i}^{+}(\mathrm{MW})$ & 0 & 20 & 0 & 20 & 20 & 0 \\
$R_{i}^{-}(\mathrm{MW})$ & 0 & 30 & 0 & 30 & 40 & 0 \\
$C_{i}^{+}(\$ / \mathrm{MWh})$ & - & 27 & - & 22 & 40 & - \\
$C_{i}^{-}(\$ / \mathrm{MWh})$ & - & 24 & - & 18 & 34 & - \\
\hline
\end{tabular}

cess to all cross-border resources. Mathematically, this organizational model is cast as a single optimization problem solved by a central entity aiming to minimize the total expected system cost. The optimal day-ahead schedule and the deployed reserves are shown in Table III, where it can be observed that wind power dispatch is equal to the low scenario. Conventional units G2, G3 and G4 are dispatched at full capacity while units G1 and G6 are in partial load due to anticipated real-time network congestion in the tie-line (3-6) if the high scenario materializes. However, unit G5 is scheduled at $40 \mathrm{MW}$ in the day-ahead stage, despite its high operational cost, to enable $40 \mathrm{MW}$ of down-regulation and accommodate the excess wind power production in scenario high.

Setup 2: This is a decentralized organizational model where the two regional operators fully coordinate on their day-ahead decisions, but there are various sources of imperfect real-time coordination. Both regional markets are cleared following the stochastic integrated market paradigm. From a mathematical viewpoint, this setup is formulated as a stochastic MCP where each operator's optimization problem, is represented by the corresponding KKT conditions. In particular, Setup $2 A$ assumes imperfect inter-regional coordination in real-time in terms of: $i$ ) no access to cross-border balancing resources and ii) no information about the domestic network of the neighboring regions, i.e., each operator is responsible only for its internal network feasibility. According to the results presented in Table III, the main differences compared to Setup 1 are found on the wind power schedule, which is now equal to the high scenario, and on the dispatch of unit G5, which is zero since no down-regulation is needed in this case. The deviation from the optimal dispatch is due to the limited information of operator II regarding the impact of scheduling the wind farm at full capacity, on the balancing operation of region I. This decision decreases the dayahead cost but in turn it deteriorates the social welfare, since the system has to resort to very costly load shedding if scenario low occurs in order to cover the deficit of $40 \mathrm{MW}$, given that only $20 \mathrm{MW}$ of up-regulation are available from unit G5. The latter is also a consequence of imperfect inter-regional coordination since any other source of flexibility is depleted already from the day-ahead stage, considering that flexible units G2 and G4 are now fully dispatched from operator II through the common day-ahead pool. However, it should be noted that operator II still has to bear some balancing costs, i.e., up-regulation of unit G5 and load shedding at node 6 , due to the physical interconnections, despite that the imbalance occurs solely within the internal network of region I.

Aiming to improve the efficiency of the previous market arrangement, we consider Setup $2 B$ where we additionally intro- 
TABLE III

MARKET OUTCOMES FOR DIFFERENT ORgANIZATIONAL SETUPS

\begin{tabular}{|c|c|c|c|c|c|c|c|c|c|}
\hline \multicolumn{2}{|r|}{ Setup1 } & \multicolumn{2}{|c|}{ Setup2A } & \multicolumn{2}{|c|}{ Setup2B } & \multicolumn{2}{|c|}{ Setup2C } & \multicolumn{2}{|c|}{ Setup3 } \\
\hline $\begin{array}{c}\text { Day-ahead } \\
\text { Schedule [MW] }\end{array}$ & Regions I \& II & Region I & Region II & Region I & Region II & Region I & Region II & Region I & Region II \\
\hline G1 & 42.2 & 21.1 & 21.1 & 29.7 & 29.7 & $50.6 / 8.6$ & $50.6 / 8.6$ & 17.2 & 17.2 \\
\hline $\mathrm{G} 2$ & 50 & 0 & 50 & 15 & 15 & $0 / 0$ & $0 / 50$ & 15 & 15 \\
\hline G3 & 50 & 0 & 50 & 25 & 25 & $5.1 / 0$ & $31.1 / 50$ & 25 & 25 \\
\hline G4 & 100 & 100 & 0 & 50 & 50 & $100 / 100$ & $0 / 0$ & 50 & 50 \\
\hline G5 & 40 & 0 & 0 & 0 & 0 & $0 / 0$ & $0 / 0$ & 5.8 & 5.8 \\
\hline G6 & 67.8 & 33.9 & 33.9 & 35.3 & 35.3 & $36.3 / 92.9$ & $36.3 / 0$ & 50 & 50 \\
\hline WP & 10 & 0 & 50 & 0 & 50 & $0 / 0$ & $50 / 50$ & 0 & 34 \\
\hline $\begin{array}{c}\text { Real-time } \\
\text { Re-dispatch } \\
\text { Actions [MW] }\end{array}$ & $r_{i_{5}, \omega_{1}}^{-}=40$ & \multicolumn{2}{|c|}{$\begin{array}{l}r_{i_{5}, \omega_{2}}^{+}=20 \\
l_{n_{3}, \omega_{2}}^{\mathrm{sh}}=8.9 \\
l_{n_{6}, \omega_{2}}^{\mathrm{sh}}=11.1\end{array}$} & \multicolumn{2}{|c|}{$\begin{array}{l}r_{i_{2}, \omega_{2}}^{+}=20 \\
r_{i_{5}, \omega_{2}}^{+}=20\end{array}$} & \multicolumn{2}{|c|}{$\begin{array}{c}r_{i_{2}, \omega_{2}}^{+}=20 / 0 \\
r_{i_{5}, \omega_{2}}^{+}=20 / 20 \\
l_{n_{3}, \omega_{2}}^{h}=0 / 20\end{array}$} & \multicolumn{2}{|c|}{$\begin{aligned} r_{i_{2}, \omega_{1}}^{-}=16, r_{i_{2}, \omega_{2}}^{+} & =20 \\
l_{n_{3}, \omega_{2}}^{\mathrm{sh}}=1.6, l_{n_{6}, \omega_{2}}^{\mathrm{sh}} & =2.4\end{aligned}$} \\
\hline $\begin{array}{c}\text { Reserved } \\
\text { Capacity [MW] }\end{array}$ & - & \multicolumn{2}{|c|}{ cor } & \multicolumn{2}{|c|}{$\begin{array}{l}\bar{r}_{i_{2}}^{+}=20 \\
\bar{r}_{i_{5}}^{+}=20\end{array}$} & \multicolumn{2}{|c|}{ - } & \multicolumn{2}{|c|}{$\begin{array}{l}\bar{r}_{i_{2}}^{+}=20 \\
\bar{r}_{i_{2}}^{-}=16\end{array}$} \\
\hline \multicolumn{10}{|c|}{ Operational costs $[\$]$} \\
\hline Day-ahead & 8517.9 & \multicolumn{2}{|c|}{7117.8} & \multicolumn{2}{|c|}{7220.6} & \multicolumn{2}{|c|}{$7648.9 / 7142.8$} & \multicolumn{2}{|c|}{7788.3} \\
\hline $\begin{array}{l}\text { Expected } \\
\text { Balancing }\end{array}$ & -816 & 711.1 & 1208.9 & 216 & 320 & $216 / 1600$ & $320 / 320$ & 113.6 & 192 \\
\hline Total & 7701.9 & \multicolumn{2}{|c|}{9037.8} & \multicolumn{2}{|c|}{7756.6} & \multicolumn{2}{|c|}{$8184.9 / 9062.8$} & \multicolumn{2}{|c|}{8093.9} \\
\hline
\end{tabular}

duce operating reserves. The corresponding results shown in Table III indicate the value of this new strategic decision variable, as reflected in the notable reduction of the total expected cost, which now becomes fairly similar to the optimal benchmark. Compared to the previous arrangement and despite that wind power schedule is still equal to the high scenario, operator $\mathrm{I}$ is now able to withdraw $20 \mathrm{MW}$ of unit G2 from the day-ahead pool to be deployed as up-regulation in the low scenario, as it can anticipate the source of the imbalance. Nonetheless, this alternative schedule eliminates the need for load shedding for any realization of the uncertainty. Given that both operators employ the stochastic integrated market-clearing, operator II infers implicitly the imbalance in region I thought the physical interconnection and consequently reserves $20 \mathrm{MW}$ from unit G5.

The previous versions of Setup 2 considered a complete market of interconnection capacities where both operators assigned the same value in these resources, i.e., the value of parameter $\Phi_{n r}^{a}$ for every tie-line $(n, r)$ in (6) is identical for both operators. In Setup $2 C$ we explore different equilibria in case of an incomplete transmission market and no operating reserves. Table III summarizes the results, separated by a slash, in cases of: i) $\Phi_{36}^{\mathrm{I}}=4-\Phi_{36}^{\mathrm{II}}=1$ and ii) $\Phi_{24}^{\mathrm{I}}=4-\Phi_{24}^{\mathrm{II}}=1$, where operator I evaluates higher either the capacity of interconnection $(3,6)$ or $(2,4)$, respectively. Considering these two normalized equilibrium points, it becomes apparent that in the absence of an organized market for the interconnection capacity, the power system operation becomes completely unpredictable. In that case, the market may reach different equilibria, with either higher or lower total cost compared to a complete market (Setup 2A), but certainly more expensive than the arrangement of perfect coordination (Setup 1). It should be noted that different combinations of the parameter $\Phi_{n r}^{a}$ were evaluated during the solution procedure. For certain values of $\Phi_{n r}^{a}$, the market equilibrium remains unchanged (equal to setup 2A) while other values of $\Phi_{n r}^{a}$ may lead to infeasibility (no market equilibrium), which emphasizes further the necessity of price coordination. Here, we follow a heuristic approach to identify a solution that highlights the implications of transmission market incompleteness, while other equilibria with analogous properties may also exist.

Setup 3: Unlike the previous setups where both regional markets accomplish perfect intra-regional coordination, Setup 3 models the interaction among variant market structures. The day-ahead settlement in region I is performed based on the 'smart' stochastic integrated market whereas region II employs the 'naive' sequential market-clearing model that prevents any temporal coordination between day-ahead and balancing operations. The mathematical model of this setup involves two distinct stages that pertain to the day-ahead and balancing operation, respectively. The first stage (St1) solves the stochastic MCP which comprises the KKT conditions of the stochastic integrated market with operating reserves and the sequential design in regions I and II, respectively, assuming that operator II does not undertake any balancing action. The second stage (St2) finds the real-time equilibrium for every uncertainty realization solving a deterministic MCP, where the day-ahead schedule is an input parameter and only the re-dispatch actions are considered as variables. Both stages assume complete sharing of day-ahead resources but no access to cross-border balancing services, while each regional operator is solely responsible for the feasibility of its internal network in real-time. Considering the results shown in Table III, it is interesting to underline that wind power is now scheduled at its expected production ( $34 \mathrm{MW}$ ), equal to the upper bound used in the sequential market-clearing of region II. Moreover, operator I who can still foresee the potential imbalances, acts proactively and procures $20 \mathrm{MW}$ and $16 \mathrm{MW}$ of up- and down-regulation respectively from unit $\mathrm{G} 2$, while the rest of the conventional generators follow the merit-order. Nonetheless, if the low scenario materializes, unit G2 will increase its production by 20 MW but still both regions will have to curtail a fraction of their demand, summing up to $4 \mathrm{MW}$, in order to maintain system balance since no additional up-regulation reserves are available. 


\section{CONCLUSIONS AND Future Work}

This paper provides a methodological framework to study different market designs with varying degrees of coordination both in the inter- and intra-regional domains. The setups of imperfect coordination, cast as generalized Nash equilibrium models, are numerically solved as stochastic MCP. The analysis performed on a stylized electricity system indicates significant reduction of the social welfare in case of imperfect quantity and transmission coordination, compared to an optimal spatio-temporally integrated benchmark. An effective remedy to restore market efficiency is an operating reserve mechanism that enables hedging against real-time market imperfections. Contrariwise, an incomplete market for the interconnection capacity yields equilibria that not only have higher cost than the optimal benchmark but are also very susceptible to the relative valuation of the tie-lines from each operator. Finally, it is shown that different market designs can coexist between adjacent regions but at the expense of increased operational cost, which underlines the need for harmonization towards an integrated European balancing market.

Future work will focus on the development of novel solution algorithms to reduce the computational burden of the proposed methodological framework. This will enable its application to real-world test cases with a large set of generation units, the detailed network representation as well as the accurate modeling of the uncertainty with an increased number of scenarios over multiple locations. Furthermore, the model can be extended to a multi-period network-constrained electricity pool to include ramping limits or other relevant time-coupling constraints, e.g., energy storage capacities. Finally, the assumption of perfect predictive densities of stochastic power generation can be relaxed in order to evaluate the effect of inaccurate forecast information on the market outcome.

\section{APPENDIX}

The complete set of KKT conditions used in the mixed complementarity problem of the stochastic integrated market without operating reserves is provided in model (9). The optimality conditions for the sequential market-clearing can be derived by removing the constraints and the corresponding dual variables that do not appear in formulation (1)-(2).

$$
\begin{aligned}
& 0 \leq C_{i}+\sum_{n \in \mathcal{M}_{n}^{I}} \hat{\lambda}_{n}+\mu_{i}^{P^{\max }} \\
& +\sum_{\omega} \sum_{i \in \mathcal{M}_{a}^{I}}\left(\mu_{i \omega}^{P R^{+}}-\mu_{i \omega}^{P R^{-}}\right) \perp p_{i}^{a} \geq 0, \forall i, \forall a \\
& 0 \leq \sum_{n \in \mathcal{M}_{n}^{J}} \hat{\lambda}_{n}+\mu_{j}^{W^{\max }}-\sum_{\omega} \sum_{n \in \mathcal{M}_{a}^{N} \cup \mathcal{M}_{n}^{J}} \hat{\lambda}_{n \omega} \perp w_{j}^{a} \geq 0, \forall j, \forall a \\
& 0=\sum_{r:(i, n) \in L} B_{n r}\left[\hat{\lambda}_{r}-\hat{\lambda}_{n}+\hat{\mu}_{n r}^{f^{\max }}-\hat{\mu}_{r n}^{\text {max }}\right. \\
& \left.+\sum_{\omega}\left(\hat{\lambda}_{n \omega}-\hat{\lambda}_{r \omega}\right)\right] \perp \hat{\delta}_{n} \text { free, }, \forall n \\
& 0 \leq \pi_{\omega} \cdot C_{i}^{+}+\sum_{n \in \mathcal{M}_{n}^{I}} \hat{\lambda}_{n \omega}+\mu_{i \omega}^{\mathrm{PR}}+\mu_{i \omega}^{\mathrm{R}^{+}} \perp r_{i \omega}^{+} \geq 0, \forall i, \forall \omega
\end{aligned}
$$

$$
\begin{aligned}
& 0 \leq-\pi_{\omega} \cdot C_{i}^{-}-\sum_{n \in \mathcal{M}_{n}^{I}} \hat{\lambda}_{n \omega}+\mu_{i \omega}^{\mathrm{PR}^{-}}+\mu_{i \omega}^{\mathrm{R}^{-}} \perp r_{i \omega}^{-} \geq 0, \forall i, \forall \omega \\
& 0 \leq \pi_{\omega} \cdot C^{\mathrm{sh}}+\hat{\lambda}_{n \omega}+\mu_{n \omega}^{l^{\mathrm{sh}}} \perp l_{n \omega}^{\mathrm{sh}} \geq 0, \forall n, \forall \omega \\
& 0 \leq-\sum_{n \in \mathcal{M}_{n}^{J}} \hat{\lambda}_{n \omega}+\mu_{j \omega}^{w^{\mathrm{spill}}} \perp w_{j \omega}^{\mathrm{spill}} \geq 0, \forall j, \forall \omega \\
& 0=\sum_{r:(i, n) \in L} B_{n r}\left[\left(\tilde{\lambda}_{r \omega}-\tilde{\lambda}_{n \omega}\right)\right. \\
&+\left.\Phi_{n r}^{a}\left(\tilde{\mu}_{n r \omega}^{f^{\max }}-\tilde{\mu}_{r n \omega}^{f^{\max }}\right)\right] \perp \tilde{\delta}_{n \omega} \text { free, }, \forall n, \forall \omega \\
& \operatorname{constraints}(1 \mathrm{~b}),(2 \mathrm{~b}),(1 \mathrm{f}),(2 \mathrm{j}) \\
& 0 \leq P_{i}^{\max }-\sum_{a} p_{i}^{a} \perp \mu_{i}^{P^{\max }} \geq 0, \forall i \\
& 0 \leq W_{j}^{\max }-\sum_{a} w_{j}^{a} \perp \mu_{j}^{W^{\max }} \geq 0, \forall j \\
& 0 \leq f_{n r}^{\max }-B_{n r}\left(\hat{\delta}_{n}-\hat{\delta}_{r}\right) \perp \hat{\mu}_{n r}^{f^{\mathrm{max}}} \geq 0, \forall(n, r) \in L \\
& 0 \leq P_{i}^{\max }-\sum_{a} p_{i}^{a}-r_{i \omega}^{+} \perp \mu_{i \omega}^{\mathrm{PR}} \geq 0, \forall i, \forall \omega \\
& 0 \leq \sum_{a} p_{i}^{a}-r_{i \omega}^{-} \perp \mu_{i \omega}^{\mathrm{PR}} \geq 0, \forall i, \forall \omega \\
& 0 \leq R_{i}^{+}-r_{i \omega}^{+} \perp \mu_{i \omega}^{\mathrm{R}^{+}} \geq 0, \forall i, \forall \omega \\
& 0 \leq R_{i}^{-}-r_{i \omega}^{-} \perp \mu_{i \omega}^{\mathrm{R}} \geq 0, \forall i, \forall \omega \\
& 0 \leq W_{j \omega}-w_{j \omega}^{\mathrm{spill}} \perp \mu_{j \omega}^{w^{\mathrm{spill}}} \geq 0, \forall j, \forall \omega \\
& 0 \leq D_{n}-l_{n \omega}^{\mathrm{sh}} \perp \mu_{n \omega}^{l^{\mathrm{sh}}} \geq 0, \forall n, \forall \omega \\
& 0 \leq f_{n r}^{\max }-B_{n r}\left(\tilde{\delta}_{n \omega}-\tilde{\delta}_{r \omega}\right) \perp \tilde{\mu}_{n r \omega}^{f_{\max }} \geq 0, \forall(n, r) \in L, \forall \omega
\end{aligned}
$$

If the operating reserves mechanism is included in the stochastic integrated market, based on the notation of Section III-D, the set of KKT conditions writes as:

$$
\begin{aligned}
& \text { constraints }(9 \mathrm{~b})-(9 \mathrm{c}),(9 \mathrm{f})-(9 \mathrm{l}),(9 \mathrm{q})-(9 \mathrm{~s}) \\
& 0 \leq C_{i}+\sum_{n \in \mathcal{M}_{n}^{I}} \hat{\lambda}_{n}+\mu_{i}^{P^{\max }} \\
& +\mu_{i}^{\mathrm{PR}^{+}}-\mu_{i}^{\mathrm{PR}^{-}} \perp p_{i}^{a} \geq 0, \forall i, \forall a \\
& 0 \leq \mu_{i}^{\mathrm{R}^{+}}+\mu_{i}^{\mathrm{PR}^{+}}-\sum_{\omega} \mu_{i \omega}^{r^{+}} \perp \bar{r}_{i}^{+} \geq 0, \forall i \\
& 0 \leq \mu_{i}^{\mathrm{R}^{-}}+\mu_{i}^{\mathrm{PR}^{-}}-\sum_{\omega} \mu_{i \omega}^{\bar{r}^{-}} \perp \bar{r}_{i}^{-} \geq 0, \forall i \\
& 0 \leq \pi_{\omega} \cdot C_{i}^{+}+\sum_{n \in \mathcal{M}_{n}^{I}} \hat{\lambda}_{n \omega}^{I}+\mu_{i \omega}^{\bar{r}^{+}} \perp r_{i \omega}^{+} \geq 0, \forall i, \forall \omega \\
& 0 \leq-\pi_{\omega} \cdot C_{i}^{-}-\sum_{n \in \mathcal{M}_{n}^{I}} \hat{\lambda}_{n \omega}+\mu_{i \omega}^{\bar{r}^{-}} \perp r_{i \omega}^{-} \geq 0, \forall i, \forall \omega \\
& 0 \leq P_{i}^{\mathrm{max}}-\sum_{a} p_{i}^{a}-\bar{r}_{i}^{+} \perp \mu_{i}^{\mathrm{PR}} \geq 0, \forall i \\
& 0 \leq \sum_{a} p_{i}^{a}-\bar{r}_{i}^{-} \perp \mu_{i}^{\mathrm{PR}} \geq 0, \forall i \\
& 0 \leq R_{i}^{+}-\bar{r}_{i}^{+} \perp \mu_{i}^{\mathrm{R}^{+}} \geq 0, \forall i \\
& 0 \leq R_{i}^{-}-\bar{r}_{i}^{-} \perp \mu_{i}^{\mathrm{R}^{-}} \geq 0, \forall i \\
& 0 \leq \bar{r}_{i}^{+}-r_{i \omega}^{+} \perp \mu_{i \omega}^{\bar{r}^{+}} \geq 0, \forall i, \forall \omega \\
& 0 \leq \bar{r}_{i}^{-}-r_{i \omega}^{-} \perp \mu_{i \omega}^{\bar{r}_{i}^{-}} \geq 0, \forall i, \forall \omega
\end{aligned}
$$




\section{ACKNOWLEDGMENT}

The authors would like to thank the editor and the four anonymous referees for their constructive comments as well as their valuable suggestions that certainly increased the value of this paper.

\section{REFERENCES}

[1] Agency for the Cooperation of Energy Regulators (ACER), Framework Guidelines on Capacity Allocation and Congestion Management for Electricity Network, Tech. Rep., 2011.

[2] P. N. Biskas, D. I. Chatzigiannis, G. A. Dourbois, and A. G. Bakirtzis, "European market integration with mixed network representation schemes," IEEE Trans. Power Syst., vol. 28, no. 4, pp. 4957-4967, Nov. 2013.

[3] P. N. Biskas, D. I. Chatzigiannis, and A. G. Bakirtzis, "European electricity market integration with mixed market designs-Part I : Formulation," IEEE Trans. Power Syst., vol. 29, no. 1, pp. 458-465, Jan. 2014

[4] H. Farahmand and G. Doorman, "Balancing market integration in the Northern European continent," Appl. Energy, vol. 96, pp. 316-326, 2012.

[5] R. A. C. van der Veen, A. Abbasy, and R. A. Hakvoort, "Analysis of the impact of cross-border balancing arrangements for Northern Europe," in Proc. 8th Int. Conf. Eur. Energy Mark. EEM 2011, 2011, pp. 653-658.

[6] S. Jaehnert and G. L. Doorman, "Assessing the benefits of regulating power market integration in Northern Europe," Elect. Power Energy Syst., vol. 43, no. 1, pp. 70-79, 2012.

[7] L. Vandezande, M. Saguan, L. Meeus, J.-M. Glachant, and R. Belmans, "Assessment of the implementation of cross-border balancing trade between Belgium and the Netherlands," in Proc. 6th Int. Conf. Eur. Energy Mark. EEM 2009, 2009, pp. 1-6.

[8] D. Newbery, G. Strbac, and I. Viehoff, The Benefits of Integrating European Electricity Markets. Cambridge, U.K.: Fac. Economics, Univ. Cambridge, 2015.

[9] G. Pritchard, G. Zakeri, and A. Philpott, "A single-settlement, energyonly electric power market for unpredictable and intermittent participants," Oper. Res., vol. 58, no. 4, pp. 1210-1219, 2010.

[10] J. M. Morales, A. J. Conejo, K. Liu, and J. Zhong, "Pricing electricity in pools with wind producers," IEEE Trans. Power Syst., vol. 27, no. 3, pp. 1366-1376, Aug. 2012.

[11] F. D. Galiana, F. Bouffard, J. M. Arroyo, and J. F. Restrepo, "Scheduling and pricing of coupled energy and primary, secondary, and tertiary reserves," Proc. IEEE, vol. 93, no. 11, pp. 1970-1982, Nov. 2005.

[12] A. Papavasiliou, S. S. Oren, and R. P. O'Neill, "Reserve requirements for wind power integration: A scenario-based stochastic programming framework," IEEE Trans. Power Syst., vol. 26, no. 4, pp. 2197-2206, Nov. 2011.

[13] R. A. C. van der Veen, A. Abbasy, and R. A. Hakvoort, "A qualitative analysis of main cross-border balancing arrangements," in Proc. 7th Int. Conf. Eur. Energy Mark. EEM 2010, 2010, pp. 1-6.

[14] K. Neuhoff, C. Batlle, G. Brunekreeft, C. Vasilakos Konstantinidis, C. Nabe, G. Oggioni, P. Rodilla, S. Schwenen, T. Siewierski, and G. Strbac, "Flexible short-term power trading: Gathering experience in EU countries," DIW Berlin Discussion Paper, 2015.

[15] G. Oggioni and Y. Smeers, "Degrees of coordination in market coupling and counter-trading," Energy J., vol. 33, no. 3, 2012.

[16] G. Oggioni, Y. Smeers, E. Allevi, and S. Schaible, "A generalized Nash equilibrium model of market coupling in the European power system," Netw. Spat. Econ., vol. 12, no. 4, pp. 503-560, 2011.

[17] G. Oggioni and Y. Smeers, "Market failures of market coupling and counter-trading in Europe: An illustrative model based discussion," Energy Econ., vol. 35, pp. 74-87, 2013.

[18] F. Kunz and A. Zerrahn, "The benefit of coordinating congestion management in Germany," in Proc. 10th Int. Conf. Eur. Energy Mark. EEM 2013, 2013, pp. 1-8.

[19] J. M. Morales, M. Zugno, S. Pineda, and P. Pinson, "Electricity market clearing with improved scheduling of stochastic production," Eur. J. Oper. Res., vol. 235, no. 3, pp. 765-774, 2014.

[20] R. Wilson, "Architecture of power markets," Econometrica, vol. 70, no. 4, pp. 1299-1340, 2002.
[21] Y. Smeers, "Market incompleteness in regional electricity transmission. Part I: The forward market," Netw. Spat. Econ., vol. 3, no. 2, pp. 175-196, 2003.

[22] M. Magill and M. Quinzii, Theory of Incomplete Markets. Cambridge, MA, USA: MIT Press, 2002.

[23] J. B. Rosen, "Existence and uniqueness of equilibrium points for concave N-person games," Econometrica, vol. 33, no. 3, pp. 520-534, 2012.

[24] K. Nabetani, P. Tseng, and M. Fukushima, "Parametrized variational inequality approaches to generalized Nash equilibrium problems with shared constraints," Comput. Optim. Appl., vol. 48, no. 3, pp. 423-452, 2009.

[25] D. Huppmann and J. Egerer, "National-strategic investment in European power transmission capacity," DIW Berlin Discussion Paper, 2014.

[26] J. Contreras, M. Klusch, and J. B. Krawczyk, "Numerical solutions to Nash-Cournot equilibria in coupled constraint electricity markets," IEEE Trans. Power Syst., vol. 19, no. 1, pp. 195-206, Feb. 2004.

[27] F. Nasiri and G. Zaccour, "Renewable portfolio standard policy: A game-theoretic analysis," INFOR, vol. 48, no. 4, pp. 251-260, 2010.

[28] C. Weber, "Adequate intraday market design to enable the integration of wind energy into the European power systems," Energy Policy, vol 38 , no. 7, pp. 3155-3163, 2010.

[29] P. T. Harker, "Generalized Nash games and quasi-variational inequalities," Eur. J. Oper. Res., vol. 54, no. 1, pp. 81-94, 1991.

[30] B. Wang and B. F. Hobbs, "A flexible ramping product: Can it help real-time dispatch markets approach the stochastic dispatch ideal?," Elect. Power Syst. Res., vol. 109, pp. 128-140, 2014.

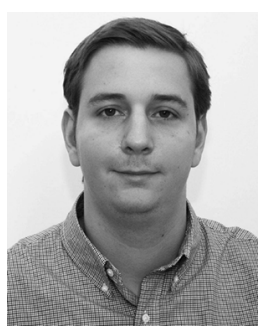

Stefanos Delikaraoglou ( $\left.\mathrm{S}^{\prime} 14\right)$ received the Dipl.-Eng. degree from the School of Mechanical Engineering, National Technical University of Athens, Greece, in 2010 and the M.Sc. degree in Sustainable Energy from the Technical University of Denmark in 2012. He is currently pursuing the Ph.D. degree at the Department of Electrical Engineering of the Technical University of Denmark.

His research interests include electricity market modeling, stochastic programming, equilibrium models and hierarchical optimization.

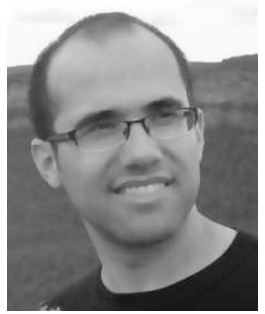

Juan M. Morales (S'07-M'11) received the Ingeniero Industrial degree from the Universidad de Málaga, Málaga, Spain, in 2006, and a Ph.D. degree in Electrical Engineering from the Universidad de Castilla-La Mancha, Ciudad Real, Spain, in 2010. He is currently an associate professor in the Department of Applied Mathematics and Computer Science at the Technical University of Denmark, Kgs. Lyngby, Denmark.

His research interests are in the fields of power systems economics, operations and planning; energy analytics and smart grids; decision-making under uncertainty, and electricity markets.

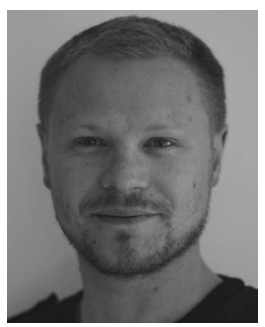

Pierre Pinson (M'11-SM'13) received the M.Sc. degree in applied mathematics from the National Institute for Applied Sciences (INSA Toulouse, France) and the Ph.D. degree in energetics from Ecole des Mines de Paris (France).

$\mathrm{He}$ is a Professor at the Technical University of Denmark, Centre for Electric Power and Energy, Department of Electrical Engineering, also heading a group focusing on Energy Analytics \& Markets. His research interests include among others forecasting, uncertainty estimation, optimization under uncertainty, decision sciences, and renewable energies.

Prof. Pinson acts as an Editor for the IEEE TRANSACTIONS ON POWER SYSTEMS, for the International Journal of Forecasting, and for Wind Energy. 


\title{
Impact of Inter- and Intra-Regional Coordination in Markets With a Large Renewable Component
}

\author{
Stefanos Delikaraoglou, Student Member, IEEE, Juan M. Morales, Member, IEEE, and \\ Pierre Pinson, Senior Member, IEEE
}

\begin{abstract}
The establishment of the single European day-ahead market has accomplished a crucial step towards the spatial integration of the European power system. However, this new arrangement does not consider any intra-regional coordination of dayahead and balancing markets and thus may become counterproductive or inefficient under uncertain supply, e.g., from weatherdriven renewable power generation. In the absence of a specific target model for the common balancing market in Europe, we introduce a framework to compare different coordination schemes and market organizations. The proposed models are formulated as stochastic equilibrium problems and compared against an optimal market setup. The simulation results reveal significant efficiency loss in case of partial coordination and diversity of market structure among regional power systems.
\end{abstract}

Index Terms-Market coupling, TSO coordination, stochastic complementarity models, generalized Nash equilibrium (GNE), electricity markets.

\section{NOTATION}

\section{A. Indices and sets:}

$A \quad$ Set of regions

$N \quad$ Set of system buses

I Set of dispatchable units

$J \quad$ Set of wind power units

$L \quad$ Set of transmission lines

$\Omega \quad$ Set of wind power scenarios

$\mathcal{M}_{n}^{I} \quad$ Set of dispatchable units located at bus $n$

$\mathcal{M}_{n}^{J} \quad$ Set of wind power units located at bus $n$

$\mathcal{M}_{a}^{N} \quad$ Set of buses located in region $a$

$\mathcal{M}_{a}^{I} \quad$ Set of dispatchable units located in region $a$

\section{B. Parameters:}

$D_{n} \quad$ Demand at node $n$ [MW]

$C_{i} \quad$ Day-ahead offer price of unit $i$ [\$/MWh]

Manuscript received September 14, 2015; revised January 02, 2016; accepted February 03, 2016. The work of S. Delikaraoglou was supported by Energinet.dk in support of ERA-Net SmartGrids through project BPES-Balancing Power in the European System (No. 2010-1-10816). The work of J. M. Morales and P. Pinson was supported in part by the Danish Strategic Council for Strategic Research through the project $5 \mathrm{~s}$ - Future Electricity Markets (no. 12-132636/DSF). Paper no. TPWRS-01302-2015.

S. Delikaraoglou and P. Pinson are with the Department of Electrical Engineering, Technical University of Denmark, Lyngby 2800, Denmark (e-mail: stde@dtu.dk; ppin@dtu.dk).

J. M. Morales is with the Department of Applied Mathematics and Computer Science, Technical University, Lyngby 2800, Denmark (e-mail: jmmgo@dtu. $\mathrm{dk})$.

$\begin{array}{ll}C_{i}^{+} & \text {Up regulation offer price of unit } i[\$ / \mathrm{MWh}] \\ C_{i}^{-} & \text {Down regulation offer price of unit } i[\$ / \mathrm{MWh}] \\ C^{\mathrm{sh}} & \text { Value of involuntarily shed load [\$/MWh] } \\ P_{i}^{\max } & \text { Capacity of dispatchable unit } i[\mathrm{MW}] \\ W_{j}^{\max } & \text { Capacity of wind power unit } j[\mathrm{MW}] \\ \bar{W}_{j} & \text { Forecast mean of wind power production [MW] } \\ W_{j \omega} & \text { Wind power realization in scenario } \omega[\mathrm{MW}] \\ \pi_{\omega} & \text { Probability of occurrence of scenario } \omega \\ R_{i}^{+} & \text {Maximum up regulation provided by unit } i \text { [MW] } \\ R_{i}^{-} & \text {Maximum down regulation provided by unit } i \\ f_{n r}^{\max } & \text { [MW] } \\ B_{n r} & \text { Maximum capacity of line }(n, r)[\mathrm{MW}] \\ \Phi_{n r}^{a} & \text { Absolute value of the susceptance of line }(n, r)\end{array}$

C. Variables:

$\hat{\delta}_{n} \quad$ Voltage angle at node $n$ at the day-ahead stage [rad]

$\tilde{\delta}_{n \omega} \quad$ Voltage angle at node $n$ in scenario $\omega$ [rad]

$p_{i} \quad$ Day-ahead schedule of dispatchable unit $i$ [MW]

$w_{j} \quad$ Day-ahead schedule of wind power unit $j$ [MW]

$\bar{r}_{i}^{+} \quad$ Up reserve capacity scheduled for unit $i$ [MW]

$\bar{r}_{i}^{-} \quad$ Down reserve capacity scheduled for unit $i$ [MW]

$r_{i \omega}^{+} \quad$ Up regulation provided by unit $i$ in scenario $\omega$ [MW]

$r_{i \omega}^{-} \quad$ Down regulation provided by unit $i$ in scenario $\omega$ [MW]

$w_{j \omega}^{\text {spill }} \quad$ Wind power spilled by unit $j$ in scenario $\omega[\mathrm{MW}]$

$l_{n \omega}^{\mathrm{sh}} \quad$ Load shedding at node $n$ in scenario $\omega$ [MW]

Variables augmented with the superscript $a$ represent the decisions of the operator in charge of the corresponding region. The symbols $p r_{A}^{\circledast}, p r_{B}^{\circledast}$ denote the valuation of the arbitrary resource $\circledast$ by operators A and B, respectively. Similarly, $q_{A}, q_{B}$ denote the subset of system resources located in the corresponding regions $\mathrm{A}$ and $\mathrm{B}$.

\section{INTRODUCTION}

$\mathbf{T}$ HE last years have seen a substantial restructuring of the European power system regarding both the generation portfolio and the market architecture. Renewable energy sources (RES) have gained a leading role on the supply-side, Digital Object Identifier 10.1109/TPWRS.2016.2530981 
while the establishment of the Internal Electricity Market (IEM) pursued the spatial integration of the regional day-ahead markets. This inter-regional integration of day-ahead markets has enabled access to a larger pool of generating resources and exploits the arbitrage opportunities over the European network to optimize social welfare through enhanced cross-border competition and improved market liquidity. According to the 'Target Model' for the European market integration, day-ahead clearing follows a zonal pricing scheme, where transmission capacity among zones is calculated according to either flow-based methods in case of highly meshed grids and strong interdependencies between the interconnections or the Available Transfer Capacity (ATC) approach for less meshed networks [1]. This reduced grid representation, where internal network is treated as a copper plate, may result into infeasible real-time power flows due to transmission congestion. Nevertheless, recent literature [2], [3] proposes alternative approaches to account also for the intra-zonal network feasibility, based on a centralized market-splitting algorithm that integrates diverse market designs, i.e., power pools or power exchanges.

Despite the spatial coupling of day-ahead markets operated by power exchanges, balancing operation remains still a responsibility of regional Transmission System Operators (TSOs). During real-time operation, TSOs must take corrective actions to guarantee network feasibility and cover any imbalances from the initial schedule. This geographically segmented design hinders access to cheaper cross-border balancing resources and thus it aggravates the scope for exercising market power in cases of low market liquidity. Moreover, internal balancing resources might be over-utilized since some potential gains from reduced prediction errors due to spatial smoothing effects of stochastic in-feed are not leveraged. In this respect, [4] shows that the integration of the Nordic and German balancing markets can reduce annual costs up to $€ 400$ million, while [5] estimates a reduction of balancing costs up to $50 \%$ from the establishment of a common merit-order list between the Dutch, German and Nordic markets. In addition, [6] reports 40\% less re-dispatch in the integrated Northern European power market in case of system-wide regulating reserve procurement as well as $25 \%$ reduction in reserves activation due to system-wide netting of imbalances. A similar analysis is performed in [7], showing a potential cost reduction of $40 \%$ by the implementation of a cross-border balancing mechanism between Belgium and the Netherlands. On the European level, [8] estimates the benefits from balancing market integration to $€ 1.3$ billion per year due to potential imbalance netting and exchange of lower cost balancing power.

Apart from the limited coordination in space, the sequential operation of the current market architecture does not allow for any inter-temporal hedging to deal with the increasing uncertainty of variable and partially predictable RES, e.g., wind power, resulting in diminished overall market efficiency. In order to enable the coupling of day-ahead and balancing market stages, recent research [9]-[12] focuses on market-clearing models that jointly optimize day-ahead dispatch and real-time reserve deployment within a stochastic programming framework. This integrated approach is able to capture the dependency of balancing actions on day-ahead decisions and thus reduce total operating costs.

Nonetheless, the implementation of an ideal market design, which integrates energy and transmission both in time and space, requires a pan-European market operator/TSO that has full information on the spatio-temporal structure of forecast errors and a complete overview of the system. However, regional TSOs are hesitant to forfeit some of their autonomy to a central authority given the significant differences that exist in balancing operations, control concepts and local resources. In the absence of a specific target model for the integration of regional balancing markets, different market arrangements with varying levels of harmonization and coordination between TSOs may arise before the establishment of a pan-European balancing market [13]. A comprehensive study on the different design elements in six short-term, i.e., intraday and real-time, European power markets is presented in [14]. In this regard, generalized Nash equilibrium (GNE) problems provide a natural framework to model the interaction of players that share a common strategy set. For instance, [15] formulates a GNE model to study different degrees of coordination in congestion management among neighboring TSOs. This model is applied on an illustrative power system in [16], [17] showing the detrimental effects of partial coordination and market incompleteness on re-dispatching costs, while [18] follows a similar approach to study the effect of imperfect coordination among the four German TSOs which results in expensive remedial actions.

This paper aims at appraising the benefits from the spatio-temporal coupling of the European electricity markets compared to plausible decentralized organizations with imperfect coordination among neighboring regions. In this regard, we provide a methodological framework to model varying levels of coordination both in space and in time based on a stochastic mixed complementarity problem (MCP) formulation. The proposed framework can accommodate several features of possible market arrangements such as limited access to cross-border balancing resources and different valuation of the shared transmission capacity. In light of an inefficient energy-only market in case of partial coordination, we propose a remedial reserve capacity mechanism that brings the market outcome close to the ideal setup of full coordination.

The rest of the paper is organized as follows. Section II describes the different degrees of intra- and inter-regional coordination and their modeling approach from an economic and mathematical perspective, while the detailed model formulation is provided in Section III. Section IV presents and discusses results of an illustrative case study performed on a stylized six-bus electricity system. Finally, Section V concludes the paper.

\section{BASIC COORDINATION CONCEPTS}

In this work, we study the impact of different degrees of coordination both in time (intra-regional) and in space (inter-regional) domains, as shown in Fig. 1.

On the temporal dimension of market operation, we model the extreme cases of imperfect and full intra-regional coordination by employing a sequential and a stochastic integrated market- 


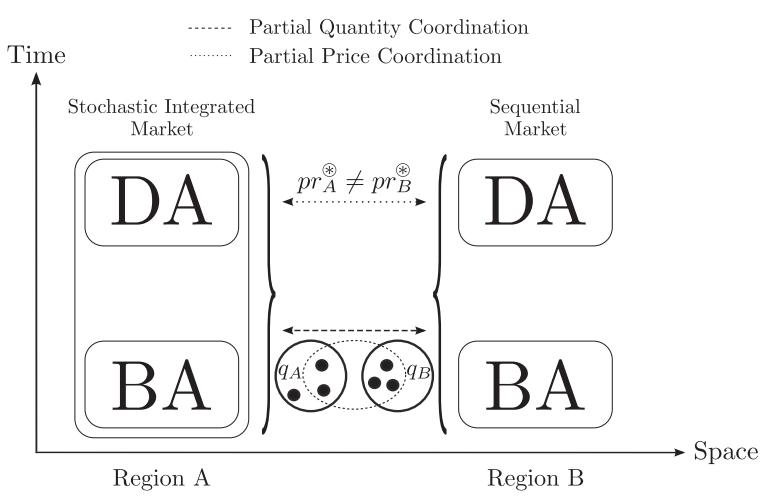

Fig. 1. Intra- and inter-regional coordination arrangements. DA: Day-ahead market. BA: Balancing market.

clearing approach, respectively. The sequential setup resembles the existing European market architecture, where a day-ahead auction takes place 12-36 hours prior to actual energy delivery to produce an initial schedule for generation and consumption levels. To cope with any real-time energy imbalances, an independent balancing market is organized for the activation of regulating power. This mechanism perceives a completely deterministic view of the uncertainties involved in power system operation, e.g., stochastic wind power generation, and thus it prevents any intra-regional coordination between the day-ahead and balancing trading floors. On the contrary, a market-clearing model which co-optimizes the energy dispatch and the anticipated reserve deployment with a fully probabilistic view of uncertainty, attains perfect intra-regional coordination. Nonetheless, this market setup suffers from a fundamental design flaw: marginal prices do not always guarantee cost recovery for flexible producers [10], [19]. Hence, the application of this market architecture here is purely instrumental and used only for the definition of an optimal benchmark for intra-regional coordination.

In the inter-regional domain, full spatial coordination is achieved when regional operators ${ }^{1}$ cooperate to jointly optimize their systems, offering all their resources in a common pool and exchanging complete information about their domestic network. On the other hand, imperfect coordination between multiple operators may arise in form of partial quantity or price coordination. Partial quantity coordination emerges in case of a segmented market of cross-border resources, i.e., when some internal resources are excluded from the common pool, as shown in Fig. 1, where only a subset of resources $q_{A}$ is offered in the common market, e.g., due to different reliability criteria. Throughout this work, we consider that all system resources are accessible from all regional operators during the day-ahead stage, following the paradigm of the European Internal Electricity Market. On the contrary, we apply the concept of partial quantity coordination on the balancing stage, where each operator can access only the subset of system resources that are physically located in its region.

\footnotetext{
${ }^{1}$ We couple the entities of market operator and TSO under the term 'operator', despite their distinguished scope pertaining to the day-ahead and balancing markets, respectively.
}

Another source of imperfect coordination is market incompleteness [20]. A complete market suggests that all constraints are traded at a single price which reflects the common valuation of each product from all agents. However, in case of partial price coordination (or a completely missing market for some services), agents with different willingness to pay for a certain resource face a price gap due to lack of arbitrage opportunities that prevent price convergence [21]. For example, in Fig. 1 the arbitrary resource $\circledast$ (e.g., a transmission line or generation capacity) is valued at different prices $p r_{A}^{\circledast} \neq p r_{B}^{\circledast}$ from each operator. This leads to a Pareto-inefficient resource allocation since all agents could improve their payoff by jointly optimizing their decisions [22].

From an economic perspective, imperfect coordination among various players relates to the notion of GNE. This is the equilibrium of a non-cooperative game where both the payoff and the set of each player's constraints depends on rival players' actions. In the context of power systems, operators in adjacent regions interact on their strategy sets through the shared physical constraints imposed by the underlying network and the limits of the available resources.

From a mathematical viewpoint, a GNE problem leads to a Quasi-Variational Inequality (QVI) model which, in the general case, admits multiple solutions that arise from different valuation of the shared constraints, i.e., each agent may have different dual variables for each shared constraint. In order to obtain a numerically tractable solution, the original QVI problem is reduced to a Variational Inequality (VI) problem, using the solution concept of normalized equilibrium [23]. Finally, this VI problem can be recast as an equivalent MCP as described in Section III.

Similar methods to explore the multiple solutions to QVI problems are proposed in [24], whereas [25] formulates an iterative algorithm based on disjunctive constraints to identify different equilibria, at the expense, though, of higher computational cost. The normalized equilibrium technique is also used in [26] to analyze cooperative game theory in the context of electricity markets as well as in [27] for the analysis of renewable portfolio standards in the presence of coupling regulatory constraints among different electricity producers.

\section{Modeling InTRA- AND INTER-REgIONAL COORDINATION}

\section{A. Assumptions}

The mathematical formulation of market-clearing models (1)-(2) and (3) build on the following set of assumptions.

1) The only source of uncertainty considered is stochastic wind power generation while system load and 'firm' resource availability can be perfectly forecast. However, additional uncertainties such as demand variations or equipment failures can be integrated in the market-clearing in a similar way.

2) Stochastic wind power production is modeled by a finite set of scenarios. This probabilistic information is centrally obtained by the operator to capture the spatial dependence structure of the forecast errors. In addition, we assume access to the correct description of the predictive densities of stochastic power production, i.e., there is no mismatch 
between the 'estimated' and the 'realized' distributions of wind energy generation. We leave the topic of forecast imperfection for future research.

3) Demand-side is assumed to be completely inelastic, with a sufficiently large value of lost load. Hence, the social welfare maximization is equivalent to minimization of the power system operational cost.

4) The day-ahead market is settled on independent hourly single-period auctions and thus no inter-temporal constraints, e.g., ramping rates, are taken into account. Nonetheless, ramping constraints can be incorporated in the proposed framework, extending the formulation to a multi-period setup. In addition, no intra-day energy trading is considered, justified by the low liquidity of these markets [28].

5) The transmission network is modeled with a lossless DC approximation.

6) Assuming null power production costs, the price offer and the spillage cost of wind power is set equal to zero.

7) In accordance with the European market paradigm, conventional generators internalize any discrete on-off commitment decisions and we further assume that they offer their full capacity into the market with linear cost functions. This preserves the linear and convex formulation of the market-clearing models and permits to integrate the market-clearing models in a complementarity problem through their Karush-Kuhn-Tucker (KKT) conditions.

In the subsequent formulations, the vector of dual variables associated with each set of constraints is indicated after a colon. For the sake of clarity, we use the symbols $\mu$ and $\lambda$, with appropriate indices, to denote the duals of inequality and equality constraints, respectively.

\section{B. Intra-Regional Coordination}

1) Sequential Market (SeqM): The sequential market setup comprises the day-ahead and balancing trading floors which are cleared independently by solving models (1) and (2), respectively. The operator determines the optimal day-ahead dispatch $\left(p_{i}^{*}, \forall i ; w_{j}^{*}, \forall j\right)$ through the following optimization problem.

$$
\underset{\Xi^{\mathrm{DA}}}{\operatorname{Min}} \sum_{i \in I} C_{i} p_{i}
$$

subject to

$$
\begin{aligned}
& \sum_{i \in \mathcal{M}_{n}^{I}} p_{i}+\sum_{j \in \mathcal{M}_{n}^{J}} w_{j}-D_{n} \\
& -\sum_{r:(n, r) \in L} B_{n r}\left(\hat{\delta}_{n}-\hat{\delta}_{r}\right)=0: \hat{\lambda}_{n}, \forall n \\
& p_{i} \leq P_{i}^{\max }: \mu_{i}^{P^{\max }}, \forall i \\
& w_{j} \leq \bar{W}_{j}: \mu_{j}^{W^{\max }}, \forall j \\
& B_{n r}\left(\hat{\delta}_{n}-\hat{\delta}_{r}\right) \leq f_{n r}^{\max }: \hat{\mu}_{n r}^{f^{\max }}, \forall(n, r) \in L \\
& \hat{\delta}_{n}=0: \hat{\lambda}^{\mathrm{ref}}, n: \operatorname{ref} \\
& p_{i} \geq 0, \forall i ; w_{j} \geq 0, \forall j ; \hat{\delta}_{n} \text { free, } \forall n \backslash n: \text { ref }
\end{aligned}
$$

where $\Xi^{\mathrm{DA}}=\left\{p_{i}, \forall i ; w_{j}, \forall j ; \hat{\delta}_{n}, \forall n\right\}$ is the set of primal optimization variables of the day-ahead market-clearing problem. The objective function (1a) represents the day-ahead cost of power system operation to be minimized. The equality constraint (1b) enforces the power balance at every node of the system. Constraints (1c) represent the capacity limits of conventional generators, while constraints (1d) set the upper bound to wind power dispatch equal to the conditional expectation of the wind power stochastic process. The power flows over the network are restricted by the transmission capacity limits in (1e) and equation (1f) fixes the voltage angle at the reference node equal to zero. Finally, constraints $(1 \mathrm{~g})$ are variable declarations.

In real-time operation, when the uncertainty realization $W_{j \omega^{\prime}}$ is known, any deviations from the day-ahead schedule have to be balanced by appropriate re-dispatch actions given as the solution of the following optimization problem.

$$
\operatorname{Min}_{\Xi^{\mathrm{RT}}} \sum_{i \in I}\left(C_{i}^{+} r_{i \omega^{\prime}}^{+}-C_{i}^{-} r_{i \omega^{\prime}}^{-}\right)+\sum_{n \in N} C^{\mathrm{sh}} l_{n \omega^{\prime}}^{\mathrm{sh}}
$$

subject to

$$
\begin{aligned}
& \sum_{i \in \mathcal{M}_{n}^{I}}\left(r_{i \omega^{\prime}}^{+}-r_{i \omega^{\prime}}^{-}\right)+\sum_{j \in \mathcal{M}_{n}^{J}}\left(W_{j \omega^{\prime}}-w_{j}^{*}-w_{j \omega^{\prime}}^{\mathrm{spill}}\right)+l_{n \omega^{\prime}}^{\mathrm{sh}} \\
& +\sum_{r:(n, r) \in L} B_{n r}\left(\hat{\delta}_{n}^{*}-\tilde{\delta}_{n \omega^{\prime}}-\hat{\delta}_{r}^{*}+\tilde{\delta}_{r \omega^{\prime}}\right)=0: \tilde{\lambda}_{n \omega^{\prime}}, \forall n(2 \mathrm{~b}) \\
& r_{i \omega^{\prime}}^{+} \leq P_{i}^{\mathrm{max}}-p_{i}^{*}: \mu_{i \omega^{\prime}}^{\mathrm{PR}^{+}}, \forall i \\
& r_{i \omega^{\prime}}^{-} \leq p_{i}^{*}: \mu_{i \omega^{\prime}}^{\mathrm{PR}}, \forall i \\
& r_{i \omega^{\prime}}^{+} \leq R_{i}^{+}: \mu_{i \omega^{\prime}}^{\mathrm{R}^{+}}, \forall i \\
& r_{i \omega^{\prime}}^{-} \leq R_{i}^{-}: \mu_{i \omega^{\prime}}^{\mathrm{R}}, \forall i \\
& w_{j \omega^{-}}^{\mathrm{spill}} \leq W_{j \omega^{\prime}}: \mu_{j \omega^{\prime}}^{w^{\mathrm{spill}}}, \forall j \\
& l_{n \omega^{\prime}}^{\mathrm{sh}} \leq D_{n}: \mu_{n \omega^{\prime}}^{l^{\mathrm{sh}}}, \forall n \\
& B_{n r}\left(\tilde{\delta}_{n \omega^{\prime}}-\tilde{\delta}_{r \omega^{\prime}}\right) \leq f_{n r}^{\max }: \tilde{\mu}_{n r \omega^{\prime}}^{f^{\max }, \forall(n, r) \in}(2 \mathrm{c}) \\
& \tilde{\delta}_{n \omega^{\prime}}=0: \tilde{\lambda}_{\omega^{\prime}}^{\mathrm{ref}}, n: \text { ref } \\
& r_{i \omega^{\prime}}^{+}, r_{i \omega^{\prime}}^{-} \geq 0, \forall i ; w_{j \omega^{\prime}}^{\mathrm{spill}} \geq 0, \forall j ; \\
& l_{n \omega^{\prime}}^{\mathrm{sh}} \geq 0, \forall n ; \tilde{\delta}_{n \omega^{\prime}} \text { free, } \forall n \backslash n: \text { ref }
\end{aligned}
$$

where $\Xi^{\mathrm{RT}}=\left\{r_{i \omega^{\prime}}^{+}, r_{i \omega^{\prime}}^{-}, \forall i ; w_{j \omega^{\prime}}^{\text {spill }}, \forall j ; l_{n \omega^{\prime}}^{\text {sh }}, \forall n ; \tilde{\delta}_{n \omega^{\prime}}, \forall n\right\}$ is the set of primal optimization variables of the balancing marketclearing problem. Notice that all the day-ahead variables enter the problem as parameters. The re-dispatch actions, i.e., up- and down-regulation, wind spillage and load shedding constitute the set of available regulation services. The objective function (2a) to be minimized is the cost of re-dispatch actions in order to re-establish the nodal power balance enforced by constraints (2b). Constraints (2c) and (2d) bind the up and down reserve activation from every conventional unit according to its capacity and the day-ahead dispatch, while constraints (2e) and (2f) enforce the corresponding offer limits specified by each generator for up- and down-regulation, respectively. Similarly, the re-dispatch actions of wind spillage and load shedding are bounded by the actual wind power production and the nodal demand through $(2 \mathrm{~g})$ and $(2 \mathrm{~h})$, respectively. Constraints (2i) guarantee the network feasibility after the deployment of reserves and equation (2j) fixes the voltage angle of the reference node to zero. The variable declarations of model (2) are included in the set of constraints (2k). 
2) Stochastic Integrated Market (StochM): The stochastic integrated market is formulated as a two-stage stochastic programming problem (3) in which day-ahead dispatch decisions anticipate the balancing recourse actions over a plausible range of uncertainty. For the sake of brevity, we present a compact formulation of model (3) emphasizing its main differences compared to the sequential approach.

$$
\underset{\Xi^{\mathrm{ST}}}{\operatorname{Min}} \sum_{i \in I} C_{i} p_{i}+\sum_{\omega \in \Omega} \pi_{\omega}\left[\sum_{i \in I}\left(C_{i}^{+} r_{i \omega}^{+}-C_{i}^{-} r_{i \omega}^{-}\right)+\sum_{n \in N} C^{\mathrm{sh}} l_{n \omega}^{\mathrm{sh}}\right]
$$

subject to

$$
\begin{aligned}
& \text { constraints }(1 \mathrm{~b})-(1 \mathrm{c}),(1 \mathrm{e})-(1 \mathrm{~g}) \\
& w_{j} \leq W_{j}^{\max }: \mu_{j}^{W^{\max }}, \forall j \\
& \text { constraints }(2 \mathrm{~b})-(2 \mathrm{k}), \forall \omega
\end{aligned}
$$

where $\Xi^{\mathrm{ST}}=\left\{p_{i}, r_{i \omega}^{+}, r_{i \omega}^{-}, \forall i, \forall \omega ; w_{j}, w_{j \omega}^{\mathrm{spill}}, \forall j, \forall \omega ; l_{n \omega}^{\mathrm{sh}}, \forall n\right.$, $\left.\forall \omega ; \hat{\delta}_{n}, \tilde{\delta}_{n \omega}, \forall n, \forall \omega\right\}$ is the set of primal optimization variables of the stochastic integrated market model. Unlike the conventional dispatch model (1)-(2), which separates completely the day-ahead and balancing operations, the stochastic integrated market accounts for the interaction of these stages through the expectation of the balancing costs in the objective function (3a) and the real-time constraints (3d). Notice that real-time constraints are formulated for all $\omega \in \Omega$, whereas the day-ahead dispatch decisions enter the set of (3d) as variables instead of fixed parameters. This implicit link between the two stages permits to pre-position, even out of merit-order, the flexible resources in a way that improves the overall response to variations and uncertainty of stochastic generation. Moreover, observe that, the day-ahead dispatch of stochastic generation may take values within the whole interval of the installed capacity (constraint (3c)) depending on the structure of forecast errors and the asymmetry of regulating costs.

\section{Inter-Regional Coordination}

To study different degrees of inter-regional coordination, we consider a multi-regional power system where each region $a \in$ $A$ is managed by an independent operator. In this setup, full coordination is achieved when all operators cooperate in order to minimize the total system cost by fully sharing the resources and technical information of their jurisdiction both in the dayahead and real-time stages. In practice, this setup is equivalent to a fully centralized organization where market-clearing is performed by a single entity. However, a decentralized organizational structure, where each regional operator seeks to minimize its own internal system cost, may not fulfill the prerequisites of the above ideal paradigm. Following the discussion of Section II, this operational scheme is modeled as a stochastic $\mathrm{MCP}$, where the KKT conditions for each operator's optimization problem are simultaneously solved. This modeling framework allows to consider regional operators that follow either similar or different market designs, in particular the 'naive' sequential approach or the 'smart' stochastic integrated marketclearing. The complete formulation of KKT optimality conditions is provided in the Appendix.
We first focus on imperfect quantity coordination only in the balancing stage, assuming that operators fully cooperate in the day-ahead stage, similarly to the existing IEM organization where all regions have access to a common system-wide pool of generation resources. Mathematically, this is implemented by replacing constraints (1c)-(1d) by (4a)-(4b), where the day-ahead dispatch variables for conventional and wind power units are now augmented with the superscript ' $a$ ' to denote the decisions of the corresponding operator. Note that in principle these are shared constraints between all operators. However, the establishment of a common day-ahead market implies that there exists only a single price for each resource, i.e., the corresponding dual variables are equal for every operator.

$$
\begin{aligned}
& \sum_{a \in A} p_{i}^{a} \leq P_{i}^{\max }: \mu_{i}^{P^{\max }}, \forall i \\
& \sum_{a \in A} w_{j}^{a} \leq \bar{W}_{j}: \mu_{j}^{W^{\max }}, \forall j
\end{aligned}
$$

Similarly, if the stochastic integrated market is employed, constraint (4b) replaces (3c) and thus its right-hand side is set equal to $W_{j}^{\max }$.

On the other hand, we assume a completely segmented balancing market where each operator has no access to cross-border resources during real-time operation. From a modeling perspective, this setup requires the replacement of reserve deployment constraints (2c)-(2d) by (5a)-(5b), which are however included only in the optimization problem of the operator who controls the region where the corresponding resource is located. Hence, these are not shared constraints, even though they include day-ahead dispatch decisions from other regional operators, and thus there exists per se a single vector of dual variables. A similar logic applies for the rest of re-dispatch actions which are solely performed by each regional operator, i.e., constraints $(2 \mathrm{e})-(2 \mathrm{~h})$. Observe that this set of constraints' substitution applies both to the sequential and the stochastic integrated market-clearing models.

$$
\begin{aligned}
& \sum_{a \in A} p_{i}^{a}+r_{i \omega}^{+} \leq P_{i}^{\mathrm{max}}: \mu_{i \omega}^{\mathrm{PR}^{+}}, \forall i \in \mathcal{M}_{a}^{I}, \forall \omega \\
& r_{i \omega}^{-}-\sum_{a \in A} p_{i}^{a} \leq 0: \mu_{i \omega}^{\mathrm{PR}^{-}} \forall i \in \mathcal{M}_{a}^{I}, \forall \omega
\end{aligned}
$$

Extending our framework into the concept of partial price coordination, we consider an incomplete market of transmission resources during balancing operation. This organizational arrangement implies that each operator is responsible to maintain the real-time power balance only in its internal network, while it has no information on the domestic grid of the neighboring regions, i.e., there is no market of internal transmission resources. Nonetheless, adjacent power systems still interact via the shared power flow constraints of the tie-lines which are influenced by the re-dispatch actions in both regions due to the underlying network topology. Hence, the transmission capacity constraints of an interconnection $(n, r)$, similar to (2i), are included in the optimization problems of both operators $a$ and $a^{\prime}$ who control the respective sending and receiving ends. As a result, there exist two distinct dual variables $\tilde{\mu}_{n r \omega}^{f^{\max } \mid a}$ and $\tilde{\mu}_{n r \omega}^{f^{\max } \mid a^{\prime}}$, reflecting the marginal valuation of each operator, which in the 
absence of a trading mechanism may not converge to a single price. In fact, this absence of a unique price for the linking constraints indicates an incomplete market of common resources and yields an under-determined system. The reason is that each player's strategic decisions influence the optimal choices of the rivals and thus the right-hand side of the shared constraints; consequently, the initial strategy has to be revised. This mathematically translates into a non-square system with more variables than equations, given that each shared constraint is complementary to two dual variables.

In order to obtain a well-defined model, [29] suggests to exogenously impose identical dual variables of the linking constraints for all players. In turn, this reduces the original GNE to a Nash equilibrium (NE) which can be modeled as a VI problem and reformulated as an equivalent $\mathrm{MCP}$, which can be readily solved. However, this numerical approach introduces an implicit coordination structure akin to a marketplace where all agents can trade their shared constraints at a common price. Considering though that we are mainly interested to model the lack of arbitrage opportunities in an incomplete market, we employ an alternative technique referred as normalized equilibrium [23]; that is, the dual variables of the shared constraints for different agents follow a predetermined linear relationship that imposes their proportional variation. This exogenous relationship reduces the degrees of freedom of the system but it prevents them from converging on the same price. A normalized equilibrium point for the tie-line capacity constraints (2i) is defined as:

$$
\tilde{\mu}_{n r \omega}^{f^{\max } \mid a}=\Phi_{n r}^{a} \circ \tilde{\mu}_{n r \omega}^{f^{\max }}, \forall(n, r) \in L, \forall \omega, \forall a,
$$

where $\circ$ denotes the element-by-element multiplication. The entries of matrix $\Phi_{n r}^{a}$ reflect the relative valuation of each line $(n, r)$ by the regional operator $a$ with respect to a common dual variable $\tilde{\mu}_{n r \omega}^{f^{\max }}$. Hence, an instance of equal $\Phi_{n r}^{a}$ elements for every operator indicates a complete market for congestion on line $(n, r)$ or a case of partial price coordination otherwise.

This method allows us to investigate distinct preferences of individual players on common resources, e.g., tie-line capacity. These preferences are expressed through the corresponding value of the parameter $\Phi_{n r}^{a}$, which reflects the perception of operator $a$ for the value of tie-line $(n, r)$. For instance, the operator of a poorly interconnected power system may rely more on a specific tie-line to ensure adequate balancing resources than the neighboring operator who is well interconnected with several other regions. Here, it should be emphasized that this mathematical implementation allows us to obtain some of the possibly multiple equilibria and does not guarantee exhaustive enumeration of all equilibrium points that may exist. Nonetheless, the focus of this work is mainly on the practical implications of incomplete markets as a form of imperfect coordination.

\section{Operating Reserves (OR)}

The stochastic integrated market of model (3) is an energy-only auction, since operational cost is proportional only to the amount of energy (day-ahead schedule and re-dispatch actions) actually delivered to the network. However, this mechanism can incorporate an additional capacity component, similar to the operating reserve markets, in order to simulate a regional electricity market that optimally draws local capacity resources from the common day-ahead pool according to the potential balancing needs to hedge against optimization errors [30]. To improve clarity, we underline the difference between operating reserves and regulation services (see also [9] and [10]). The former term refers to the procurement of reserve $\mathrm{ca}$ pacity which, in turn, is excluded from energy provision in the day-ahead market. On the other hand, regulation services relate to the actual deployment of operating reserves during real-time operation. These re-dispatch actions modify the day-ahead production/consumption schedule of the generators/loads and thus, provide an energy service to the system.

With the introduction of the operating reserves mechanism, the amount of capacity reserved in each regional market becomes an additional strategic decision variable of the corresponding operator, denoted by $\bar{r}_{i}^{+}$and $\bar{r}_{i}^{-}$for up and down reserve capacity, respectively. For this purpose, we add the dayahead constraints $(7 a)-(7 b)$ that bound the upward and downward reserve provision to the respective capacity offer and constraints (7c)-(7d) that define the complementary relationship of energy and reserve services.

$$
\begin{aligned}
& \bar{r}_{i}^{+} \leq R_{i}^{+}: \mu_{i}^{\mathrm{R}^{+}}, \forall i \\
& \bar{r}_{i}^{-} \leq R_{i}^{-}: \mu_{i}^{\mathrm{R}^{-}}, \forall i \\
& \sum_{a \in A} p_{i}^{a}+\bar{r}_{i}^{+} \leq P_{i}^{\mathrm{max}}: \mu_{i}^{\mathrm{PR}^{+}}, \forall i \\
& \bar{r}_{i}^{-}-\sum_{a \in A} p_{i}^{a} \leq 0: \mu_{i}^{\mathrm{PR}^{-}}, \forall i
\end{aligned}
$$

In the balancing counterpart, constraints (2c)-(2d) are removed, since they now become redundant, and constraints (2e)-(2f) are replaced by

$$
\begin{aligned}
& r_{i \omega}^{+} \leq \bar{r}_{i}^{+}: \mu_{i \omega}^{\bar{r}^{+}}, \forall i, \forall \omega \\
& r_{i \omega}^{-} \leq \bar{r}_{i}^{-}: \mu_{i \omega}^{\bar{r}^{-}}, \forall i, \forall \omega .
\end{aligned}
$$

Following a similar process, operating reserves can be integrated also in the sequential market-clearing model (1)-(2). However, this would require the definition of explicit reserve requirements, which is beyond the scope of this paper. In addition, we would like to underline that operating reserves are not perceived here as a tradable product with explicit reserve capacity costs, but as a tool to indicate divergent policies for sharing internal balancing resources. Nonetheless, flexible generators are allowed to submit regulation offers with price premiums that compensate the opportunity costs of the power capacity withdrawn from the day-ahead dispatch, following the approach of [9].

\section{CASE Study}

The main purpose of this section is to provide some qualitative insights regarding the effect of imperfect coordination between neighboring power systems. To this aim, we consider the three distinct organizational setups presented in Fig. 2, with varying degrees of coordination and/or market architecture. The 


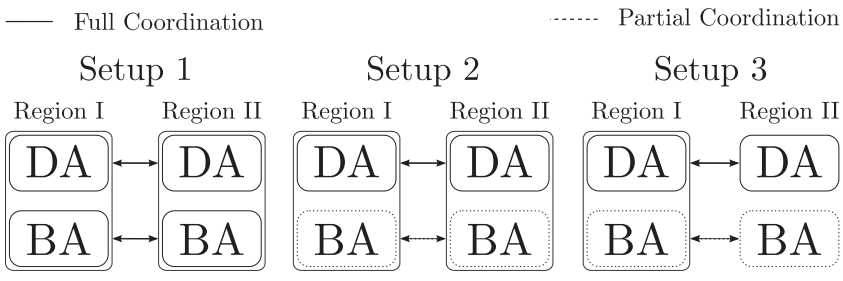

Fig. 2. Schematic representation of organizational setups with different market designs and degrees of coordination.

TABLE I

Model Definition for Organizational Setups

\begin{tabular}{llcc}
\hline Setup & \multicolumn{1}{c}{$\begin{array}{c}\text { Market } \\
\text { Organization }\end{array}$} & $\begin{array}{c}\text { Model } \\
\text { Definition }\end{array}$ & $\Phi_{n r}^{a}$ \\
\hline 1 & StochM & (3a)-(3d) & - \\
\hline 2A & StochM & $(9 \mathrm{a})-(9 \mathrm{~s})$ & $\Phi_{n r}^{a}=1$ \\
2B & StochM + OR & $(10 \mathrm{a})-(101)$ & $\Phi_{n r}^{a}=1$ \\
2C & StochM & $(9 \mathrm{a})-(9 \mathrm{~s})$ & $\Phi_{n r}^{a} \neq \Phi_{n r}^{a^{\prime}}$ \\
\hline 3 & Region I: StochM + OR & Stl: $(10 \mathrm{a})-(101) \dagger$ & \multirow{2}{*}{$\Phi_{n r}^{a}=1$} \\
\hline
\end{tabular}

† Without variables pertaining to the balancing actions of region II.

${ }_{\ddagger}^{\ddagger}$ Solved for each scenario with the day-ahead variables fixed to $S t l$.

SeqM: Sequential Market. StochM: Stochastic Integrated Market. OR: Operating Reserves.

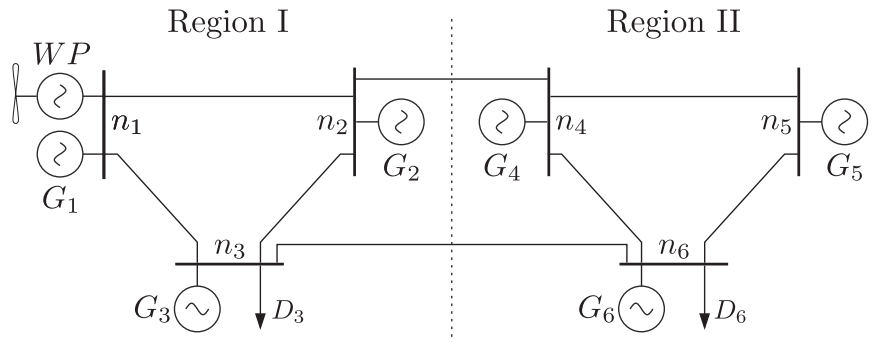

Fig. 3. Six-node power system.

model definition and the market architecture for each setup are summarized in Table I.

We consider the six-bus system depicted in Fig. 3 which comprises two regions. The transmission capacities and the reactances of all domestic lines are equal to 0.13 p.u. and $100 \mathrm{MW}$, respectively. The corresponding values for the tie-lines connecting region I to region II, are equal to 0.20 p.u. and $15 \mathrm{MW}$. Each region includes both flexible and inflexible conventional generators (G1 to G6) and one wind power plant (WP) is located at node 1 . The stochastic wind power generation is modeled using two scenarios, namely high $\left(\omega_{1}: 50 \mathrm{MW}\right)$ and low $\left(\omega_{2}\right.$ : $10 \mathrm{MW}$ ), with a probability of occurrence 0.6 and 0.4 , respectively. The quantity and the price offers of conventional units for the day-ahead and balancing markets are provided in Table II. In addition, two inelastic loads (D3 and D6) of $170 \mathrm{MW}$ and $190 \mathrm{MW}$, placed at nodes 3 and 6 , can be partially curtailed at a cost, $C^{\text {sh }}$, of $\$ 200 / \mathrm{MWh}$.

Setup 1: For our analysis, Setup 1 corresponds to perfect coordination both in the inter- and intra-regional domains. Here, both operators employ the stochastic integrated market design and fully cooperate in order to ensure system-wide network feasibility both in day-ahead and real-time operation with full ac-
TABLE II

Generator Data

\begin{tabular}{lcccccc}
\hline Unit $i$ & 1 & 2 & 3 & 4 & 5 & 6 \\
\hline$P_{i}^{\max }(\mathrm{MW})$ & 110 & 50 & 50 & 100 & 100 & 100 \\
$C_{i}(\$ / M W h)$ & 30 & 25 & 10 & 20 & 35 & 31 \\
$R_{i}^{+}(\mathrm{MW})$ & 0 & 20 & 0 & 20 & 20 & 0 \\
$R_{i}^{-}(\mathrm{MW})$ & 0 & 30 & 0 & 30 & 40 & 0 \\
$C_{i}^{+}(\$ / \mathrm{MWh})$ & - & 27 & - & 22 & 40 & - \\
$C_{i}^{-}(\$ / \mathrm{MWh})$ & - & 24 & - & 18 & 34 & - \\
\hline
\end{tabular}

cess to all cross-border resources. Mathematically, this organizational model is cast as a single optimization problem solved by a central entity aiming to minimize the total expected system cost. The optimal day-ahead schedule and the deployed reserves are shown in Table III, where it can be observed that wind power dispatch is equal to the low scenario. Conventional units G2, G3 and G4 are dispatched at full capacity while units G1 and G6 are in partial load due to anticipated real-time network congestion in the tie-line (3-6) if the high scenario materializes. However, unit G5 is scheduled at $40 \mathrm{MW}$ in the day-ahead stage, despite its high operational cost, to enable $40 \mathrm{MW}$ of down-regulation and accommodate the excess wind power production in scenario high.

Setup 2: This is a decentralized organizational model where the two regional operators fully coordinate on their day-ahead decisions, but there are various sources of imperfect real-time coordination. Both regional markets are cleared following the stochastic integrated market paradigm. From a mathematical viewpoint, this setup is formulated as a stochastic MCP where each operator's optimization problem, is represented by the corresponding KKT conditions. In particular, Setup $2 A$ assumes imperfect inter-regional coordination in real-time in terms of: $i$ ) no access to cross-border balancing resources and ii) no information about the domestic network of the neighboring regions, i.e., each operator is responsible only for its internal network feasibility. According to the results presented in Table III, the main differences compared to Setup 1 are found on the wind power schedule, which is now equal to the high scenario, and on the dispatch of unit G5, which is zero since no down-regulation is needed in this case. The deviation from the optimal dispatch is due to the limited information of operator II regarding the impact of scheduling the wind farm at full capacity, on the balancing operation of region I. This decision decreases the dayahead cost but in turn it deteriorates the social welfare, since the system has to resort to very costly load shedding if scenario low occurs in order to cover the deficit of $40 \mathrm{MW}$, given that only $20 \mathrm{MW}$ of up-regulation are available from unit G5. The latter is also a consequence of imperfect inter-regional coordination since any other source of flexibility is depleted already from the day-ahead stage, considering that flexible units G2 and G4 are now fully dispatched from operator II through the common day-ahead pool. However, it should be noted that operator II still has to bear some balancing costs, i.e., up-regulation of unit G5 and load shedding at node 6 , due to the physical interconnections, despite that the imbalance occurs solely within the internal network of region I.

Aiming to improve the efficiency of the previous market arrangement, we consider Setup $2 B$ where we additionally intro- 
TABLE III

MARKET OUTCOMES FOR DIFFERENT ORgANIZATIONAL SETUPS

\begin{tabular}{|c|c|c|c|c|c|c|c|c|c|}
\hline \multicolumn{2}{|r|}{ Setup1 } & \multicolumn{2}{|c|}{ Setup2A } & \multicolumn{2}{|c|}{ Setup2B } & \multicolumn{2}{|c|}{ Setup2C } & \multicolumn{2}{|c|}{ Setup3 } \\
\hline $\begin{array}{c}\text { Day-ahead } \\
\text { Schedule [MW] }\end{array}$ & Regions I \& II & Region I & Region II & Region I & Region II & Region I & Region II & Region I & Region II \\
\hline G1 & 42.2 & 21.1 & 21.1 & 29.7 & 29.7 & $50.6 / 8.6$ & $50.6 / 8.6$ & 17.2 & 17.2 \\
\hline $\mathrm{G} 2$ & 50 & 0 & 50 & 15 & 15 & $0 / 0$ & $0 / 50$ & 15 & 15 \\
\hline G3 & 50 & 0 & 50 & 25 & 25 & $5.1 / 0$ & $31.1 / 50$ & 25 & 25 \\
\hline G4 & 100 & 100 & 0 & 50 & 50 & $100 / 100$ & $0 / 0$ & 50 & 50 \\
\hline G5 & 40 & 0 & 0 & 0 & 0 & $0 / 0$ & $0 / 0$ & 5.8 & 5.8 \\
\hline G6 & 67.8 & 33.9 & 33.9 & 35.3 & 35.3 & $36.3 / 92.9$ & $36.3 / 0$ & 50 & 50 \\
\hline WP & 10 & 0 & 50 & 0 & 50 & $0 / 0$ & $50 / 50$ & 0 & 34 \\
\hline $\begin{array}{c}\text { Real-time } \\
\text { Re-dispatch } \\
\text { Actions [MW] }\end{array}$ & $r_{i_{5}, \omega_{1}}^{-}=40$ & \multicolumn{2}{|c|}{$\begin{array}{l}r_{i_{5}, \omega_{2}}^{+}=20 \\
l_{n_{3}, \omega_{2}}^{\mathrm{sh}}=8.9 \\
l_{n_{6}, \omega_{2}}^{\mathrm{sh}}=11.1\end{array}$} & \multicolumn{2}{|c|}{$\begin{array}{l}r_{i_{2}, \omega_{2}}^{+}=20 \\
r_{i_{5}, \omega_{2}}^{+}=20\end{array}$} & \multicolumn{2}{|c|}{$\begin{array}{c}r_{i_{2}, \omega_{2}}^{+}=20 / 0 \\
r_{i_{5}, \omega_{2}}^{+}=20 / 20 \\
l_{n_{3}, \omega_{2}}^{h}=0 / 20\end{array}$} & \multicolumn{2}{|c|}{$\begin{aligned} r_{i_{2}, \omega_{1}}^{-}=16, r_{i_{2}, \omega_{2}}^{+} & =20 \\
l_{n_{3}, \omega_{2}}^{\mathrm{sh}}=1.6, l_{n_{6}, \omega_{2}}^{\mathrm{sh}} & =2.4\end{aligned}$} \\
\hline $\begin{array}{c}\text { Reserved } \\
\text { Capacity [MW] }\end{array}$ & - & \multicolumn{2}{|c|}{ cor } & \multicolumn{2}{|c|}{$\begin{array}{l}\bar{r}_{i_{2}}^{+}=20 \\
\bar{r}_{i_{5}}^{+}=20\end{array}$} & \multicolumn{2}{|c|}{ - } & \multicolumn{2}{|c|}{$\begin{array}{l}\bar{r}_{i_{2}}^{+}=20 \\
\bar{r}_{i_{2}}^{-}=16\end{array}$} \\
\hline \multicolumn{10}{|c|}{ Operational costs $[\$]$} \\
\hline Day-ahead & 8517.9 & \multicolumn{2}{|c|}{7117.8} & \multicolumn{2}{|c|}{7220.6} & \multicolumn{2}{|c|}{$7648.9 / 7142.8$} & \multicolumn{2}{|c|}{7788.3} \\
\hline $\begin{array}{l}\text { Expected } \\
\text { Balancing }\end{array}$ & -816 & 711.1 & 1208.9 & 216 & 320 & $216 / 1600$ & $320 / 320$ & 113.6 & 192 \\
\hline Total & 7701.9 & \multicolumn{2}{|c|}{9037.8} & \multicolumn{2}{|c|}{7756.6} & \multicolumn{2}{|c|}{$8184.9 / 9062.8$} & \multicolumn{2}{|c|}{8093.9} \\
\hline
\end{tabular}

duce operating reserves. The corresponding results shown in Table III indicate the value of this new strategic decision variable, as reflected in the notable reduction of the total expected cost, which now becomes fairly similar to the optimal benchmark. Compared to the previous arrangement and despite that wind power schedule is still equal to the high scenario, operator $\mathrm{I}$ is now able to withdraw $20 \mathrm{MW}$ of unit G2 from the day-ahead pool to be deployed as up-regulation in the low scenario, as it can anticipate the source of the imbalance. Nonetheless, this alternative schedule eliminates the need for load shedding for any realization of the uncertainty. Given that both operators employ the stochastic integrated market-clearing, operator II infers implicitly the imbalance in region I thought the physical interconnection and consequently reserves $20 \mathrm{MW}$ from unit G5.

The previous versions of Setup 2 considered a complete market of interconnection capacities where both operators assigned the same value in these resources, i.e., the value of parameter $\Phi_{n r}^{a}$ for every tie-line $(n, r)$ in (6) is identical for both operators. In Setup $2 C$ we explore different equilibria in case of an incomplete transmission market and no operating reserves. Table III summarizes the results, separated by a slash, in cases of: i) $\Phi_{36}^{\mathrm{I}}=4-\Phi_{36}^{\mathrm{II}}=1$ and ii) $\Phi_{24}^{\mathrm{I}}=4-\Phi_{24}^{\mathrm{II}}=1$, where operator I evaluates higher either the capacity of interconnection $(3,6)$ or $(2,4)$, respectively. Considering these two normalized equilibrium points, it becomes apparent that in the absence of an organized market for the interconnection capacity, the power system operation becomes completely unpredictable. In that case, the market may reach different equilibria, with either higher or lower total cost compared to a complete market (Setup 2A), but certainly more expensive than the arrangement of perfect coordination (Setup 1). It should be noted that different combinations of the parameter $\Phi_{n r}^{a}$ were evaluated during the solution procedure. For certain values of $\Phi_{n r}^{a}$, the market equilibrium remains unchanged (equal to setup 2A) while other values of $\Phi_{n r}^{a}$ may lead to infeasibility (no market equilibrium), which emphasizes further the necessity of price coordination. Here, we follow a heuristic approach to identify a solution that highlights the implications of transmission market incompleteness, while other equilibria with analogous properties may also exist.

Setup 3: Unlike the previous setups where both regional markets accomplish perfect intra-regional coordination, Setup 3 models the interaction among variant market structures. The day-ahead settlement in region I is performed based on the 'smart' stochastic integrated market whereas region II employs the 'naive' sequential market-clearing model that prevents any temporal coordination between day-ahead and balancing operations. The mathematical model of this setup involves two distinct stages that pertain to the day-ahead and balancing operation, respectively. The first stage (St1) solves the stochastic MCP which comprises the KKT conditions of the stochastic integrated market with operating reserves and the sequential design in regions I and II, respectively, assuming that operator II does not undertake any balancing action. The second stage (St2) finds the real-time equilibrium for every uncertainty realization solving a deterministic MCP, where the day-ahead schedule is an input parameter and only the re-dispatch actions are considered as variables. Both stages assume complete sharing of day-ahead resources but no access to cross-border balancing services, while each regional operator is solely responsible for the feasibility of its internal network in real-time. Considering the results shown in Table III, it is interesting to underline that wind power is now scheduled at its expected production ( $34 \mathrm{MW}$ ), equal to the upper bound used in the sequential market-clearing of region II. Moreover, operator I who can still foresee the potential imbalances, acts proactively and procures $20 \mathrm{MW}$ and $16 \mathrm{MW}$ of up- and down-regulation respectively from unit $\mathrm{G} 2$, while the rest of the conventional generators follow the merit-order. Nonetheless, if the low scenario materializes, unit G2 will increase its production by 20 MW but still both regions will have to curtail a fraction of their demand, summing up to $4 \mathrm{MW}$, in order to maintain system balance since no additional up-regulation reserves are available. 


\section{CONCLUSIONS AND Future Work}

This paper provides a methodological framework to study different market designs with varying degrees of coordination both in the inter- and intra-regional domains. The setups of imperfect coordination, cast as generalized Nash equilibrium models, are numerically solved as stochastic MCP. The analysis performed on a stylized electricity system indicates significant reduction of the social welfare in case of imperfect quantity and transmission coordination, compared to an optimal spatio-temporally integrated benchmark. An effective remedy to restore market efficiency is an operating reserve mechanism that enables hedging against real-time market imperfections. Contrariwise, an incomplete market for the interconnection capacity yields equilibria that not only have higher cost than the optimal benchmark but are also very susceptible to the relative valuation of the tie-lines from each operator. Finally, it is shown that different market designs can coexist between adjacent regions but at the expense of increased operational cost, which underlines the need for harmonization towards an integrated European balancing market.

Future work will focus on the development of novel solution algorithms to reduce the computational burden of the proposed methodological framework. This will enable its application to real-world test cases with a large set of generation units, the detailed network representation as well as the accurate modeling of the uncertainty with an increased number of scenarios over multiple locations. Furthermore, the model can be extended to a multi-period network-constrained electricity pool to include ramping limits or other relevant time-coupling constraints, e.g., energy storage capacities. Finally, the assumption of perfect predictive densities of stochastic power generation can be relaxed in order to evaluate the effect of inaccurate forecast information on the market outcome.

\section{APPENDIX}

The complete set of KKT conditions used in the mixed complementarity problem of the stochastic integrated market without operating reserves is provided in model (9). The optimality conditions for the sequential market-clearing can be derived by removing the constraints and the corresponding dual variables that do not appear in formulation (1)-(2).

$$
\begin{aligned}
& 0 \leq C_{i}+\sum_{n \in \mathcal{M}_{n}^{I}} \hat{\lambda}_{n}+\mu_{i}^{P^{\max }} \\
& +\sum_{\omega} \sum_{i \in \mathcal{M}_{a}^{I}}\left(\mu_{i \omega}^{P R^{+}}-\mu_{i \omega}^{P R^{-}}\right) \perp p_{i}^{a} \geq 0, \forall i, \forall a \\
& 0 \leq \sum_{n \in \mathcal{M}_{n}^{J}} \hat{\lambda}_{n}+\mu_{j}^{W^{\max }}-\sum_{\omega} \sum_{n \in \mathcal{M}_{a}^{N} \cup \mathcal{M}_{n}^{J}} \hat{\lambda}_{n \omega} \perp w_{j}^{a} \geq 0, \forall j, \forall a \\
& 0=\sum_{r:(i, n) \in L} B_{n r}\left[\hat{\lambda}_{r}-\hat{\lambda}_{n}+\hat{\mu}_{n r}^{f^{\max }}-\hat{\mu}_{r n}^{\text {max }}\right. \\
& \left.+\sum_{\omega}\left(\hat{\lambda}_{n \omega}-\hat{\lambda}_{r \omega}\right)\right] \perp \hat{\delta}_{n} \text { free, }, \forall n \\
& 0 \leq \pi_{\omega} \cdot C_{i}^{+}+\sum_{n \in \mathcal{M}_{n}^{I}} \hat{\lambda}_{n \omega}+\mu_{i \omega}^{\mathrm{PR}}+\mu_{i \omega}^{\mathrm{R}^{+}} \perp r_{i \omega}^{+} \geq 0, \forall i, \forall \omega
\end{aligned}
$$

$$
\begin{aligned}
& 0 \leq-\pi_{\omega} \cdot C_{i}^{-}-\sum_{n \in \mathcal{M}_{n}^{I}} \hat{\lambda}_{n \omega}+\mu_{i \omega}^{\mathrm{PR}^{-}}+\mu_{i \omega}^{\mathrm{R}^{-}} \perp r_{i \omega}^{-} \geq 0, \forall i, \forall \omega \\
& 0 \leq \pi_{\omega} \cdot C^{\mathrm{sh}}+\hat{\lambda}_{n \omega}+\mu_{n \omega}^{l^{\mathrm{sh}}} \perp l_{n \omega}^{\mathrm{sh}} \geq 0, \forall n, \forall \omega \\
& 0 \leq-\sum_{n \in \mathcal{M}_{n}^{J}} \hat{\lambda}_{n \omega}+\mu_{j \omega}^{w^{\mathrm{spill}}} \perp w_{j \omega}^{\mathrm{spill}} \geq 0, \forall j, \forall \omega \\
& 0=\sum_{r:(i, n) \in L} B_{n r}\left[\left(\tilde{\lambda}_{r \omega}-\tilde{\lambda}_{n \omega}\right)\right. \\
&+\left.\Phi_{n r}^{a}\left(\tilde{\mu}_{n r \omega}^{f^{\max }}-\tilde{\mu}_{r n \omega}^{f^{\max }}\right)\right] \perp \tilde{\delta}_{n \omega} \text { free, }, \forall n, \forall \omega \\
& \operatorname{constraints}(1 \mathrm{~b}),(2 \mathrm{~b}),(1 \mathrm{f}),(2 \mathrm{j}) \\
& 0 \leq P_{i}^{\max }-\sum_{a} p_{i}^{a} \perp \mu_{i}^{P^{\max }} \geq 0, \forall i \\
& 0 \leq W_{j}^{\max }-\sum_{a} w_{j}^{a} \perp \mu_{j}^{W^{\max }} \geq 0, \forall j \\
& 0 \leq f_{n r}^{\max }-B_{n r}\left(\hat{\delta}_{n}-\hat{\delta}_{r}\right) \perp \hat{\mu}_{n r}^{f^{\mathrm{max}}} \geq 0, \forall(n, r) \in L \\
& 0 \leq P_{i}^{\max }-\sum_{a} p_{i}^{a}-r_{i \omega}^{+} \perp \mu_{i \omega}^{\mathrm{PR}} \geq 0, \forall i, \forall \omega \\
& 0 \leq \sum_{a} p_{i}^{a}-r_{i \omega}^{-} \perp \mu_{i \omega}^{\mathrm{PR}} \geq 0, \forall i, \forall \omega \\
& 0 \leq R_{i}^{+}-r_{i \omega}^{+} \perp \mu_{i \omega}^{\mathrm{R}^{+}} \geq 0, \forall i, \forall \omega \\
& 0 \leq R_{i}^{-}-r_{i \omega}^{-} \perp \mu_{i \omega}^{\mathrm{R}} \geq 0, \forall i, \forall \omega \\
& 0 \leq W_{j \omega}-w_{j \omega}^{\mathrm{spill}} \perp \mu_{j \omega}^{w^{\mathrm{spill}}} \geq 0, \forall j, \forall \omega \\
& 0 \leq D_{n}-l_{n \omega}^{\mathrm{sh}} \perp \mu_{n \omega}^{l^{\mathrm{sh}}} \geq 0, \forall n, \forall \omega \\
& 0 \leq f_{n r}^{\max }-B_{n r}\left(\tilde{\delta}_{n \omega}-\tilde{\delta}_{r \omega}\right) \perp \tilde{\mu}_{n r \omega}^{f_{\max }} \geq 0, \forall(n, r) \in L, \forall \omega
\end{aligned}
$$

If the operating reserves mechanism is included in the stochastic integrated market, based on the notation of Section III-D, the set of KKT conditions writes as:

$$
\begin{aligned}
& \text { constraints }(9 \mathrm{~b})-(9 \mathrm{c}),(9 \mathrm{f})-(9 \mathrm{l}),(9 \mathrm{q})-(9 \mathrm{~s}) \\
& 0 \leq C_{i}+\sum_{n \in \mathcal{M}_{n}^{I}} \hat{\lambda}_{n}+\mu_{i}^{P^{\max }} \\
& +\mu_{i}^{\mathrm{PR}^{+}}-\mu_{i}^{\mathrm{PR}^{-}} \perp p_{i}^{a} \geq 0, \forall i, \forall a \\
& 0 \leq \mu_{i}^{\mathrm{R}^{+}}+\mu_{i}^{\mathrm{PR}^{+}}-\sum_{\omega} \mu_{i \omega}^{r^{+}} \perp \bar{r}_{i}^{+} \geq 0, \forall i \\
& 0 \leq \mu_{i}^{\mathrm{R}^{-}}+\mu_{i}^{\mathrm{PR}^{-}}-\sum_{\omega} \mu_{i \omega}^{\bar{r}^{-}} \perp \bar{r}_{i}^{-} \geq 0, \forall i \\
& 0 \leq \pi_{\omega} \cdot C_{i}^{+}+\sum_{n \in \mathcal{M}_{n}^{I}} \hat{\lambda}_{n \omega}^{I}+\mu_{i \omega}^{\bar{r}^{+}} \perp r_{i \omega}^{+} \geq 0, \forall i, \forall \omega \\
& 0 \leq-\pi_{\omega} \cdot C_{i}^{-}-\sum_{n \in \mathcal{M}_{n}^{I}} \hat{\lambda}_{n \omega}+\mu_{i \omega}^{\bar{r}^{-}} \perp r_{i \omega}^{-} \geq 0, \forall i, \forall \omega \\
& 0 \leq P_{i}^{\mathrm{max}}-\sum_{a} p_{i}^{a}-\bar{r}_{i}^{+} \perp \mu_{i}^{\mathrm{PR}} \geq 0, \forall i \\
& 0 \leq \sum_{a} p_{i}^{a}-\bar{r}_{i}^{-} \perp \mu_{i}^{\mathrm{PR}} \geq 0, \forall i \\
& 0 \leq R_{i}^{+}-\bar{r}_{i}^{+} \perp \mu_{i}^{\mathrm{R}^{+}} \geq 0, \forall i \\
& 0 \leq R_{i}^{-}-\bar{r}_{i}^{-} \perp \mu_{i}^{\mathrm{R}^{-}} \geq 0, \forall i \\
& 0 \leq \bar{r}_{i}^{+}-r_{i \omega}^{+} \perp \mu_{i \omega}^{\bar{r}^{+}} \geq 0, \forall i, \forall \omega \\
& 0 \leq \bar{r}_{i}^{-}-r_{i \omega}^{-} \perp \mu_{i \omega}^{\bar{r}_{i}^{-}} \geq 0, \forall i, \forall \omega
\end{aligned}
$$




\section{ACKNOWLEDGMENT}

The authors would like to thank the editor and the four anonymous referees for their constructive comments as well as their valuable suggestions that certainly increased the value of this paper.

\section{REFERENCES}

[1] Agency for the Cooperation of Energy Regulators (ACER), Framework Guidelines on Capacity Allocation and Congestion Management for Electricity Network, Tech. Rep., 2011.

[2] P. N. Biskas, D. I. Chatzigiannis, G. A. Dourbois, and A. G. Bakirtzis, "European market integration with mixed network representation schemes," IEEE Trans. Power Syst., vol. 28, no. 4, pp. 4957-4967, Nov. 2013.

[3] P. N. Biskas, D. I. Chatzigiannis, and A. G. Bakirtzis, "European electricity market integration with mixed market designs-Part I : Formulation," IEEE Trans. Power Syst., vol. 29, no. 1, pp. 458-465, Jan. 2014

[4] H. Farahmand and G. Doorman, "Balancing market integration in the Northern European continent," Appl. Energy, vol. 96, pp. 316-326, 2012.

[5] R. A. C. van der Veen, A. Abbasy, and R. A. Hakvoort, "Analysis of the impact of cross-border balancing arrangements for Northern Europe," in Proc. 8th Int. Conf. Eur. Energy Mark. EEM 2011, 2011, pp. 653-658.

[6] S. Jaehnert and G. L. Doorman, "Assessing the benefits of regulating power market integration in Northern Europe," Elect. Power Energy Syst., vol. 43, no. 1, pp. 70-79, 2012.

[7] L. Vandezande, M. Saguan, L. Meeus, J.-M. Glachant, and R. Belmans, "Assessment of the implementation of cross-border balancing trade between Belgium and the Netherlands," in Proc. 6th Int. Conf. Eur. Energy Mark. EEM 2009, 2009, pp. 1-6.

[8] D. Newbery, G. Strbac, and I. Viehoff, The Benefits of Integrating European Electricity Markets. Cambridge, U.K.: Fac. Economics, Univ. Cambridge, 2015.

[9] G. Pritchard, G. Zakeri, and A. Philpott, "A single-settlement, energyonly electric power market for unpredictable and intermittent participants," Oper. Res., vol. 58, no. 4, pp. 1210-1219, 2010.

[10] J. M. Morales, A. J. Conejo, K. Liu, and J. Zhong, "Pricing electricity in pools with wind producers," IEEE Trans. Power Syst., vol. 27, no. 3, pp. 1366-1376, Aug. 2012.

[11] F. D. Galiana, F. Bouffard, J. M. Arroyo, and J. F. Restrepo, "Scheduling and pricing of coupled energy and primary, secondary, and tertiary reserves," Proc. IEEE, vol. 93, no. 11, pp. 1970-1982, Nov. 2005.

[12] A. Papavasiliou, S. S. Oren, and R. P. O'Neill, "Reserve requirements for wind power integration: A scenario-based stochastic programming framework," IEEE Trans. Power Syst., vol. 26, no. 4, pp. 2197-2206, Nov. 2011.

[13] R. A. C. van der Veen, A. Abbasy, and R. A. Hakvoort, "A qualitative analysis of main cross-border balancing arrangements," in Proc. 7th Int. Conf. Eur. Energy Mark. EEM 2010, 2010, pp. 1-6.

[14] K. Neuhoff, C. Batlle, G. Brunekreeft, C. Vasilakos Konstantinidis, C. Nabe, G. Oggioni, P. Rodilla, S. Schwenen, T. Siewierski, and G. Strbac, "Flexible short-term power trading: Gathering experience in EU countries," DIW Berlin Discussion Paper, 2015.

[15] G. Oggioni and Y. Smeers, "Degrees of coordination in market coupling and counter-trading," Energy J., vol. 33, no. 3, 2012.

[16] G. Oggioni, Y. Smeers, E. Allevi, and S. Schaible, "A generalized Nash equilibrium model of market coupling in the European power system," Netw. Spat. Econ., vol. 12, no. 4, pp. 503-560, 2011.

[17] G. Oggioni and Y. Smeers, "Market failures of market coupling and counter-trading in Europe: An illustrative model based discussion," Energy Econ., vol. 35, pp. 74-87, 2013.

[18] F. Kunz and A. Zerrahn, "The benefit of coordinating congestion management in Germany," in Proc. 10th Int. Conf. Eur. Energy Mark. EEM 2013, 2013, pp. 1-8.

[19] J. M. Morales, M. Zugno, S. Pineda, and P. Pinson, "Electricity market clearing with improved scheduling of stochastic production," Eur. J. Oper. Res., vol. 235, no. 3, pp. 765-774, 2014.

[20] R. Wilson, "Architecture of power markets," Econometrica, vol. 70, no. 4, pp. 1299-1340, 2002.
[21] Y. Smeers, "Market incompleteness in regional electricity transmission. Part I: The forward market," Netw. Spat. Econ., vol. 3, no. 2, pp. 175-196, 2003.

[22] M. Magill and M. Quinzii, Theory of Incomplete Markets. Cambridge, MA, USA: MIT Press, 2002.

[23] J. B. Rosen, "Existence and uniqueness of equilibrium points for concave N-person games," Econometrica, vol. 33, no. 3, pp. 520-534, 2012.

[24] K. Nabetani, P. Tseng, and M. Fukushima, "Parametrized variational inequality approaches to generalized Nash equilibrium problems with shared constraints," Comput. Optim. Appl., vol. 48, no. 3, pp. 423-452, 2009.

[25] D. Huppmann and J. Egerer, "National-strategic investment in European power transmission capacity," DIW Berlin Discussion Paper, 2014.

[26] J. Contreras, M. Klusch, and J. B. Krawczyk, "Numerical solutions to Nash-Cournot equilibria in coupled constraint electricity markets," IEEE Trans. Power Syst., vol. 19, no. 1, pp. 195-206, Feb. 2004.

[27] F. Nasiri and G. Zaccour, "Renewable portfolio standard policy: A game-theoretic analysis," INFOR, vol. 48, no. 4, pp. 251-260, 2010.

[28] C. Weber, "Adequate intraday market design to enable the integration of wind energy into the European power systems," Energy Policy, vol 38 , no. 7, pp. 3155-3163, 2010.

[29] P. T. Harker, "Generalized Nash games and quasi-variational inequalities," Eur. J. Oper. Res., vol. 54, no. 1, pp. 81-94, 1991.

[30] B. Wang and B. F. Hobbs, "A flexible ramping product: Can it help real-time dispatch markets approach the stochastic dispatch ideal?," Elect. Power Syst. Res., vol. 109, pp. 128-140, 2014.

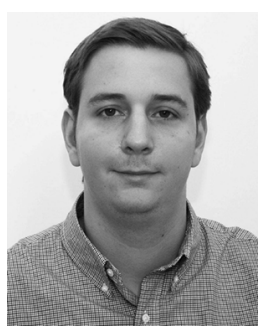

Stefanos Delikaraoglou ( $\left.\mathrm{S}^{\prime} 14\right)$ received the Dipl.-Eng. degree from the School of Mechanical Engineering, National Technical University of Athens, Greece, in 2010 and the M.Sc. degree in Sustainable Energy from the Technical University of Denmark in 2012. He is currently pursuing the Ph.D. degree at the Department of Electrical Engineering of the Technical University of Denmark.

His research interests include electricity market modeling, stochastic programming, equilibrium models and hierarchical optimization.

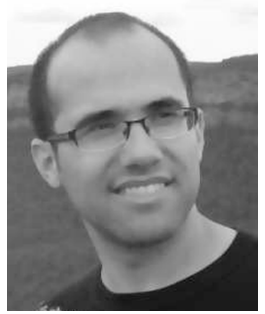

Juan M. Morales (S'07-M'11) received the Ingeniero Industrial degree from the Universidad de Málaga, Málaga, Spain, in 2006, and a Ph.D. degree in Electrical Engineering from the Universidad de Castilla-La Mancha, Ciudad Real, Spain, in 2010. He is currently an associate professor in the Department of Applied Mathematics and Computer Science at the Technical University of Denmark, Kgs. Lyngby, Denmark.

His research interests are in the fields of power systems economics, operations and planning; energy analytics and smart grids; decision-making under uncertainty, and electricity markets.

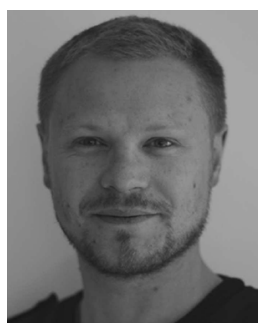

Pierre Pinson (M'11-SM'13) received the M.Sc. degree in applied mathematics from the National Institute for Applied Sciences (INSA Toulouse, France) and the Ph.D. degree in energetics from Ecole des Mines de Paris (France).

$\mathrm{He}$ is a Professor at the Technical University of Denmark, Centre for Electric Power and Energy, Department of Electrical Engineering, also heading a group focusing on Energy Analytics \& Markets. His research interests include among others forecasting, uncertainty estimation, optimization under uncertainty, decision sciences, and renewable energies.

Prof. Pinson acts as an Editor for the IEEE TRANSACTIONS ON POWER SYSTEMS, for the International Journal of Forecasting, and for Wind Energy. 